\title{
PROJECTIVE OBJECTS IN THE CATEGORY OF POINTWISE FINITE DIMENSIONAL REPRESENTATIONS OF AN INTERVAL FINITE QUIVER
}

\author{
PENGJIE JIAO
}

\begin{abstract}
For an interval finite quiver $Q$, we introduce a class of flat representations. We classify the indecomposable projective objects in the category $\operatorname{rep}(Q)$ of pointwise finite dimensional representations. We show that an object in $\operatorname{rep}(Q)$ is projective if and only if it is a direct sum of countably generated flat representations.
\end{abstract}

\section{INTRODUCTION}

Let $k$ be a field and let $Q$ be an interval finite quiver (may contain infinitely many vertices). Denote by $\operatorname{Rep}(Q)$ the category of representations of $Q$ over $k$, and by $\operatorname{rep}(Q)$ the full subcategory formed by pointwise finite dimensional representations.

We mention that infinite quivers appear naturally in the covering theory of algebras; see [5, 8. The representation theory of some infinite quivers is studied in [4]. The result is applied to the study of the bounded derived category of an algebra with radical square zero; see 3 .

We are interested in the Auslander-Reiten theory of $\operatorname{rep}(Q)$. The reason is due to that almost split sequences in $\operatorname{rep}(Q)$ seem to behave better than the ones in certain subcategories of $\operatorname{rep}(Q)$; see [4, Section 2]. Moreover, $\operatorname{rep}(Q)$ is easier to study than $\operatorname{Rep}(Q)$.

The very first step is to understand the projective objects and then the projectively trivial morphisms (see [14, Section 2]) in $\operatorname{rep}(Q)$. It is well known that each representation in $\operatorname{rep}(Q)$ is a direct sum of indecomposable representations, whose endomorphism ring is local; see Lemma 3.6. Hence, it is sufficient to study indecomposable projective objects in $\operatorname{rep}(Q)$.

In this paper, we are able to classify the indecomposable projective objects in $\operatorname{rep}(Q)$, and characterize the projective objects via flat representations of $Q$. To state the results, we introduce some notations.

For each vertex $a$, we denote by $P_{a}$ the usual indecomposable projective representation corresponding to $a$.

Let $p$ be a right infinite path, which means an infinite sequence of arrows $\alpha_{1} \alpha_{2} \cdots \alpha_{n} \cdots$ with $s\left(\alpha_{i}\right)=t\left(\alpha_{i+1}\right)$ for each $i \geq 1$. Recall that the convex hull of $p$ is the smallest convex subquiver of $Q$ containing $p$. It is called uniformly interval finite if the set of finite paths $u$ with $s(u)=a, t(u)=b$ for any given vertices $a, b$ is bounded uniformly.

Denote by $[p]$ the equivalence class (see page 3 for the definition) of right infinite paths containing $p$. We introduce a flat representation $X_{[p]}$ and show that it is an indecomposable projective object in $\operatorname{rep}(Q)$, if the convex hull of any right infinite path in $[p]$ is uniformly interval finite; see Proposition 6.2 .

Date: October 23, 2019.

2010 Mathematics Subject Classification. 16D40, 18A30, 16G20.

Key words and phrases. Interval finite quiver, flat representations, pointwise finite dimensional representations, projective objects. 
Moreover, we give a complete classification of indecomposable projective objects, and hence projective objects in $\operatorname{rep}(Q)$.

Theorem A (see Theorem 6.7). Let $Q$ be an interval finite quiver. Assume that $P$ is an indecomposable projective object in $\operatorname{rep}(Q)$. Then either $P \simeq P_{a}$ for some vertex $a$, or $P \simeq X_{[p]}$ for some equivalence class $[p]$ of right infinite paths, where the convex hull of any right infinite path in $[p]$ is uniformly interval finite.

It is well known that a finitely presented representation is projective if and only if it is flat. Inspired by this fact, we investigate the relationship between flat representations lying in $\operatorname{rep}(Q)$ and projective objects in $\operatorname{rep}(Q)$.

More precisely, we obtain the following characterization for flat representations. This strengthens the Lazard-Govorov Theorem [10, 13] in the special case $\operatorname{Rep}(Q)$.

Proposition B (see Proposition 5.2 ). A flat representation in $\operatorname{Rep}(Q)$ is a direct limit of finitely generated projective subrepresentations.

Based on this description, we can give a characterization for projective objects in terms of flat representations. This is analogous to the classical result [7, Theorem 2.2] in module categories, which is due to [12] and [16].

Theorem C (see Theorem [7.2). Let $Q$ be an interval finite quiver and let $M$ be a representation in $\operatorname{rep}(Q)$. Then $M$ is projective in $\operatorname{rep}(Q)$ if and only if $M$ is a direct sum of countably generated flat representations in $\operatorname{Rep}(Q)$.

The paper is organized as follows. In Section 2 we introduce the notion of uniformly interval finite quiver. We give characterizations for the convex hull of a right infinite path being uniformly interval finite. In Section 3 we recall some basic facts about representations of quivers. In Section 4 we recall some results about direct limits and inverse limits. In Section 5 we introduce a class of flat representations $X_{[p]}$ for each equivalence class [p] of right infinite paths. We study morphisms between representations of the form $X_{[p]}$. We give a characterization for flat representations in $\operatorname{Rep}(Q)$. Sections 6 and 7 are dedicated to the proofs of Theorems 6.7 and 7.2, respectively.

\section{UNIFORMLY INTERVAL FINITE QUIVERS}

Let $Q=\left(Q_{0}, Q_{1}\right)$ be a quiver, where $Q_{0}$ is the set of vertices and $Q_{1}$ is the set of arrows. Given an arrow $\alpha: a \rightarrow b$, we denote by $s(\alpha)=a$ its source and by $t(\alpha)=b$ its target.

A (finite) path $p$ of length $l \geq 1$ is a sequence of arrows $\alpha_{l} \cdots \alpha_{2} \alpha_{1}$ such that $s\left(\alpha_{i+1}\right)=t\left(\alpha_{i}\right)$ for each $i=1,2, \ldots, l-1$. We set $s(p)=s\left(\alpha_{1}\right)$ and $t(p)=t\left(\alpha_{l}\right)$. We associate with each vertex $a$ a trivial path (of length 0) $e_{a}$ such that $s\left(e_{a}\right)=$ $a=t\left(e_{a}\right)$. A nontrivial finite path $p$ is called an oriented cycle if $s(p)=t(p)$.

Let $a, b \in Q_{0}$. Denote by $Q(a, b)$ the set of finite paths $p$ with $s(p)=a$ and $t(p)=b$. If $Q(a, b) \neq \emptyset$, then $a$ is called a predecessor of $b$, and $b$ is called a successor of $a$. Moreover, if $Q(a, b)$ contains an arrow, then $a$ is called a direct predecessor of $b$, and $b$ is called a direct successor of $a$. Denote by $a^{+}$the set of its direct successors, and by $b^{-}$the set of its direct predecessors.

A right infinite path $p$ is an infinite sequence of arrows $\alpha_{1} \alpha_{2} \cdots \alpha_{n} \cdots$ such that $s\left(\alpha_{i}\right)=t\left(\alpha_{i+1}\right)$ for each $i \geq 1$. We set $t(p)=t\left(\alpha_{1}\right)$. Dually, a left infinite path $p$ is an infinite sequence of arrows $\cdots \alpha_{n} \cdots \alpha_{2} \alpha_{1}$ such that $s\left(\alpha_{i+1}\right)=t\left(\alpha_{i}\right)$ for each $i \geq 1$. We set $s(p)=s\left(\alpha_{1}\right)$. Here, we use the terminologies in [6, Subsection 2.1]. We mention that the pair of notions are opposite to the corresponding ones in [4, Section 1].

Recall that $Q$ is called connected if its underlying graph is connected. We call $Q$ interval finite if $Q(a, b)$ is finite for any vertices $a$ and $b$. We mention that an 
interval finite quiver contains no oriented cycles. We call $Q$ locally finite if for any vertex $a$, the set of arrows $\alpha$ with $s(\alpha)=a$ or $t(\alpha)=a$ is finite. We call $Q$ strongly locally finite for short if $Q$ is locally finite and interval finite.

The opposite quiver $Q^{\text {op }}$ of $Q$ means the quiver $\left(Q_{0}^{\mathrm{op}}, Q_{1}^{\mathrm{op}}\right)$, where $Q_{0}^{\mathrm{op}}=Q_{0}$ and $Q_{1}^{\mathrm{op}}=\left\{\alpha^{\mathrm{op}}: b \rightarrow a \mid Q_{1} \ni \alpha: a \rightarrow b\right\}$. Given a finite path $p=\alpha_{n} \cdots \alpha_{2} \alpha_{1}$ in $Q$, we set the corresponding finite path $p^{\mathrm{op}}=\alpha_{1}^{\mathrm{op}} \alpha_{2}^{\mathrm{op}} \cdots \alpha_{n}^{\mathrm{op}}$ in $Q^{\mathrm{op}}$. Similarly, given a right infinite path $p=\alpha_{1} \alpha_{2} \cdots \alpha_{n} \cdots$ and a left infinite path $q=\cdots \beta_{n} \cdots \beta_{2} \beta_{1}$ in $Q$, we set the corresponding left infinite path $p^{\mathrm{op}}=\cdots \alpha_{n}^{\mathrm{op}} \cdots \alpha_{2}^{\mathrm{op}} \alpha_{1}^{\mathrm{op}}$ and right infinite path $q^{\text {op }}=\beta_{1}^{\text {op }} \beta_{2}^{\text {op }} \cdots \beta_{n}^{\text {op }} \ldots$ in $Q^{\text {op }}$.

Following [6, Subsection 2.1], we introduce an equivalence relation on right infinite paths in $Q$. Two right infinite paths $\alpha_{1} \alpha_{2} \cdots \alpha_{i} \cdots$ and $\beta_{1} \beta_{2} \cdots \beta_{j} \cdots$ are equivalent, if there exist some positive integers $m$ and $n$ such that

$$
\alpha_{m} \alpha_{m+1} \cdots \alpha_{i} \cdots=\beta_{n} \beta_{n+1} \cdots \beta_{j} \cdots .
$$

Given a right infinite path $p$, we denote by $[p]$ the equivalence class containing $p$. For each vertex $a$, we denote by $[p]_{a}$ the subclass of $[p]$ consisting of right infinite paths $p^{\prime}$ with $t\left(p^{\prime}\right)=a$. We mention that both $[p]$ and $[p]_{a}$ are sets.

Dually, two left infinite paths $\cdots \alpha_{i} \cdots \alpha_{2} \alpha_{1}$ and $\cdots \beta_{j} \cdots \beta_{2} \beta_{1}$ are equivalent, if there exist some positive integers $m$ and $n$ such that

$$
\cdots \alpha_{i} \cdots \alpha_{m+1} \alpha_{m}=\cdots \beta_{j} \cdots \beta_{n+1} \beta_{n} .
$$

Given a left infinite path $q$, we denote by $[q]$ the equivalence class containing $q$.

Recall that a right infinite path is called cyclic, if it is of the form $u u \cdots u \cdots$ for some oriented cycle $u$. A right infinite path is called rational if it is equivalent to a cyclic right infinite path; otherwise, it is called irrational. We observe that a rational right infinite path can be written as the form $q u u \cdots u \cdots$ for some finite path $q$ and some oriented cycle $u$. Then any right infinite path is irrational if $Q$ contains no oriented cycles.

For a right infinite path $p$, we mention the following observation.

Lemma 2.1. Let $p$ be a right infinite path. Assume $q p=q^{\prime} p$ for some finite paths $q$ and $q^{\prime}$ with $s(q)=t(p)=s\left(q^{\prime}\right)$ and $q \neq q^{\prime}$. Then $p$ is cyclic.

Proof. We observe that the lengths of $q$ and $q^{\prime}$ are not the same, since $q p=q^{\prime} p$ and $q \neq q^{\prime}$. We may assume the length of $q^{\prime}$ is greater than the one of $q$. Then there exists some nontrivial finite path $u$ such that $q^{\prime}=q u$. We have that $s(u)=s\left(q^{\prime}\right)=$ $s(q)=t(u)$. In other words, $u$ is an oriented cycle. We observe that $q p=q^{\prime} p=q u p$, and then $p=u p$. It follows inductively that $p=u u \cdots u \cdots$.

Recall that a subquiver $Q^{\prime}$ of $Q$ is called full if each arrow $\alpha$ with $s(\alpha), t(\alpha) \in Q_{0}^{\prime}$ lies in $Q^{\prime}$. We call $Q^{\prime}$ convex if each finite path $p$ with $s(p), t(p) \in Q_{0}^{\prime}$ lies in $Q^{\prime}$. Given a finite path (or an infinite path) $p$, the smallest convex subquiver of $Q$ containing $p$ is called the convex hull of $p$.

Given an equivalence class $[p]$ of irrational right infinite paths, we have the following characterization of $\left|[p]_{t(p)}\right|$. Here, the symbol "|.|" means the cardinal number of a set.

Lemma 2.2. Let $p=\alpha_{1} \alpha_{2} \cdots \alpha_{i} \cdots$ be an irrational right infinite path. Denote by $\Omega$ the convex hull of $p$, and set $a_{i}=t\left(\alpha_{i+1}\right)$ for any $i \geq 0$. Then

$$
\left|[p]_{a_{0}}\right|=\sup _{a, b \in \Omega}|Q(a, b)|=\sup _{i, j \geq 0}\left|Q\left(a_{i}, a_{j}\right)\right|=\sup _{i \geq 0}\left|Q\left(a_{i}, a_{0}\right)\right| .
$$

Proof. We observe by the definition of the convex hull that for any vertices $a$ and $b$ in $\Omega$, there exist some finite paths $u \in Q\left(b, a_{0}\right)$ and $v \in Q\left(a_{i}, a\right)$ for some $i \geq 0$. The injection

$$
f: Q(a, b) \longrightarrow Q\left(a_{i}, a_{0}\right), \quad q \mapsto u q v,
$$


implies that $|Q(a, b)| \leq\left|Q\left(a_{i}, a_{0}\right)\right|$. We then obtain

$$
\sup _{i \geq 0}\left|Q\left(a_{i}, a_{0}\right)\right| \geq \sup _{a, b \in \Omega}|Q(a, b)| \geq \sup _{i, j \geq 0}\left|Q\left(a_{i}, a_{j}\right)\right| \geq \sup _{i \geq 0}\left|Q\left(a_{i}, a_{0}\right)\right| .
$$

It remains to show $\left|[p]_{a_{0}}\right|=\sup _{i \geq 0}\left|Q\left(a_{i}, a_{0}\right)\right|$.

For each $i \geq 0$, let $\Delta_{i}$ be the subset of $[p]_{a_{0}}$ consisting of right infinite paths of the form $u \alpha_{i+1} \alpha_{i+2} \cdots \alpha_{j} \cdots$ for some finite path $u$. We have that $\Delta_{i} \subseteq \Delta_{i+1}$ and $[p]_{a_{0}}=\bigcup_{i \geq 0} \Delta_{i}$. Consider the surjection

$$
g: Q\left(a_{i}, a_{0}\right) \longrightarrow \Delta_{i}, \quad q \mapsto q \alpha_{i+1} \alpha_{i+2} \cdots \alpha_{j} \cdots .
$$

By the assumption, the right infinite path $\alpha_{i+1} \alpha_{i+2} \cdots \alpha_{j} \cdots$ can not be cyclic. Then Lemma 2.1 implies that $g$ is an injection, and hence is a bijection. It follows that

$$
\left|[p]_{a_{0}}\right|=\sup _{i \geq 0}\left|\Delta_{i}\right|=\sup _{i \geq 0}\left|Q\left(a_{i}, a_{0}\right)\right| .
$$

Here, we introduce a notion stronger than interval finite.

Definition 2.3. We call a quiver uniformly interval finite if there exists some integer $N$ such that for each pair of vertices $a$ and $b$, the number of finite paths $p$ with $s(p)=a$ and $t(p)=b$ is less than or equal to $N$.

Example 2.4. (1) The infinite quivers of $A_{\infty}, A_{\infty}^{\infty}$ and $D_{\infty}$ type are uniformly interval finite.

(2) The infinite quiver of the form

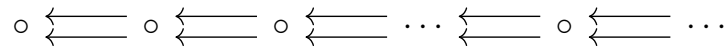

is interval finite but not uniformly interval finite.

The following result is a consequence of Lemma 2.2

Proposition 2.5. Let $p=\alpha_{1} \alpha_{2} \cdots \alpha_{i} \cdots$ be an irrational right infinite path. Set $a_{i}=t\left(\alpha_{i+1}\right)$ for each $i \geq 0$. The following statements are equivalent.

(1) The convex hull of $p$ is uniformly interval finite.

(2) $\left\{\left|Q\left(a_{i}, a_{0}\right)\right| \mid i \geq 0\right\}$ is bounded.

(3) $[p]_{a_{0}}$ is finite.

Proof. Let $\Omega$ be the convex hull of $p$. We observe that $\Omega$ being uniformly interval finite precisely means $\{|Q(a, b)| \mid a, b \in \Omega\}$ being bounded. Then it follows from Lemma 2.2 that (1), (2) and (3) are equivalent.

The following lemma gives some necessary conditions for the convex hull of a right infinite path being uniformly interval finite.

Lemma 2.6. Let $p=\alpha_{1} \alpha_{2} \cdots \alpha_{i} \cdots$ be a right infinite path, whose convex hull is uniformly interval finite. Set $a_{i}=t\left(\alpha_{i+1}\right)$ for each $i \geq 0$. Then there exists some nonnegative integer $N$ such that $\left|Q\left(a_{i}, a_{j}\right)\right|=1$ and $\left|[p]_{a_{j}}\right|=1$ for any $i \geq j \geq N$.

Proof. Since the convex hull of $p$ is uniformly interval finite, it contains no oriented cycles. In particular, $p$ is irrational. By the equivalence between Proposition 2.5(1) and Proposition 2.5 (2), we have that $\left\{\left|Q\left(a_{i}, a_{0}\right)\right| \mid i \geq 0\right\}$ is bounded. For any $i \geq$ $j \geq l \geq 0$, we have the injection

$$
f: Q\left(a_{j}, a_{l}\right) \times Q\left(a_{i}, a_{j}\right) \longrightarrow Q\left(a_{i}, a_{l}\right), \quad(u, v) \mapsto u v .
$$

We obtain $\left|Q\left(a_{i}, a_{0}\right)\right| \geq\left|Q\left(a_{j}, a_{0}\right)\right| \times\left|Q\left(a_{i}, a_{j}\right)\right| \geq\left|Q\left(a_{j}, a_{0}\right)\right|$. Then there exists some nonnegative integer $N$ such that $\left|Q\left(a_{i}, a_{0}\right)\right|=\left|Q\left(a_{N}, a_{0}\right)\right|$ for any $i \geq N$. Since $\left|Q\left(a_{N}, a_{0}\right)\right| \times\left|Q\left(a_{i}, a_{N}\right)\right| \leq\left|Q\left(a_{i}, a_{0}\right)\right|$, we have that $\left|Q\left(a_{i}, a_{N}\right)\right|=1$. Since $\left|Q\left(a_{j}, a_{N}\right)\right| \times\left|Q\left(a_{i}, a_{j}\right)\right| \leq\left|Q\left(a_{i}, a_{N}\right)\right|$ for any $i \geq j \geq N$, we have that $\left|Q\left(a_{i}, a_{j}\right)\right|=$ 1. By Lemma 2.2, we obtain $\left|[p]_{a_{j}}\right|=1$. 
We mention that the necessary conditions in the above lemma is not sufficient; see the following example.

Example 2.7. (1) Let $Q$ be the following quiver

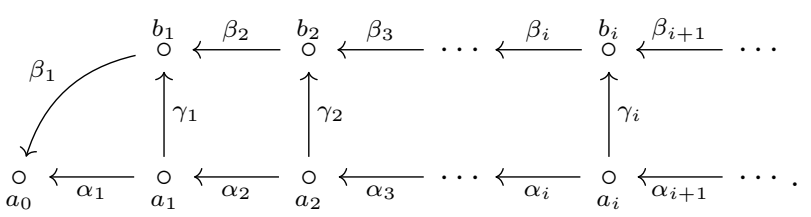

Let $\Omega$ be the set of equivalence classes of right infinite paths, and let $\Delta$ be the set of right infinite paths $p$ with $t(p)=a_{0}$.

Set $u=\alpha_{1} \alpha_{2} \cdots \alpha_{i} \cdots$ and $v=\beta_{1} \beta_{2} \cdots \beta_{i} \cdots$. For each $i \geq 1$, we set

$$
w_{i}=\beta_{1} \beta_{2} \cdots \beta_{i} \gamma_{i} \alpha_{i+1} \alpha_{i+2} \cdots \alpha_{j} \cdots
$$

Then we have that

$$
\Delta=\{u, v\} \cup\left\{w_{i} \mid i \geq 1\right\} .
$$

We observe that each class in $\Omega$ contains some right infinite path in $\Delta$. Since $[u]_{a_{0}}=\{u\} \cup\left\{w_{i} \mid i \geq 1\right\}$ and $[v]_{a_{0}}=\{v\}$, we have that

$$
\Omega=\{[u],[v]\} .
$$

We observe that $\left|Q\left(b_{i}, a_{0}\right)\right|=1$ for any $i \geq 0$. Then Proposition 2.5 implies that the convex hull of $v$ is uniformly interval finite. Moreover, the convex hull of any right infinite path in $[v]$ is uniformly interval finite.

For each $i \geq 1$, we have that $\left|Q\left(a_{i}, a_{0}\right)\right|=i+1$. Then Proposition 2.5 implies that the convex hull of $u$ is not uniformly interval finite. Similarly, neither is the one of any $w_{l}$. But $\left|Q\left(a_{i}, a_{j}\right)\right|=1$ and $\left|[p]_{a_{j}}\right|=1$ for any $i \geq j \geq 1$. Then the convex hull of each $\alpha_{j} \alpha_{j+1} \cdots \alpha_{i} \cdots$ is uniformly interval finite by Proposition 2.5.

(2) Let $Q$ be the following quiver

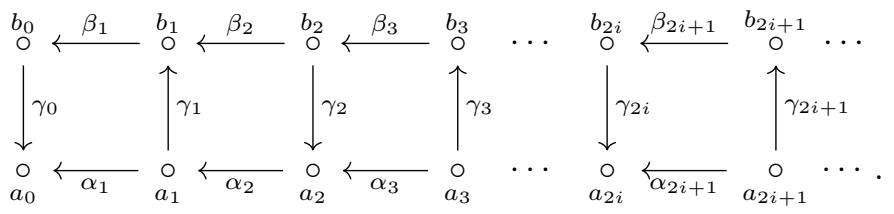

Let $\Delta$ be the set of right infinite paths $p$ with $t(p)=a_{0}$. Then $\Delta$ is uncountable. Let $\Omega$ be the set of equivalence classes of right infinite paths. We observe that each class in $\Omega$ contains at least one right infinite path in $\Delta$, and at most countably many right infinite paths in $\Delta$. Therefore $\Omega$ is uncountable.

For any positive integer $N$, we have that $\left|Q\left(a_{N+2}, a_{N}\right)\right|=2$. Then Lemma 2.6 implies that the convex hull of $\alpha_{1} \alpha_{2} \cdots \alpha_{i} \cdots$ is not uniformly interval finite.

Moreover, one can show that the convex hull of any right infinite path $p$ in $\Delta$ is not uniformly interval finite. Indeed, we observe that either $a_{i}$ or $b_{i}$ will appear in $p$ for any $i \geq 0$. For any positive integer $N$, we have that $\left|Q\left(c, c^{\prime}\right)\right| \geq 2$ for any $c \in\left\{a_{N+3}, b_{N+3}\right\}$ and $c^{\prime} \in\left\{a_{N}, b_{N}\right\}$. Then Lemma2.6 implies that the convex hull of $p$ is not uniformly interval finite. 


\section{REPRESENTATIONS OF QUiVERS}

Let $k$ be a field and let $Q$ be a quiver. Denote by $\operatorname{Mod} k$ the category of $k$-linear spaces.

A representation $M=(M(a), M(\alpha))$ of $Q$ over $k$ is given by $k$-linear spaces $M(a)$ for any vertex $a$, and $k$-linear maps $M(\alpha): M(a) \rightarrow M(b)$ for any arrow $\alpha: a \rightarrow b$. For each finite path $p=\alpha_{l} \cdots \alpha_{2} \alpha_{1}$ of length $l \geq 1$, we set $M(p)=$ $M\left(\alpha_{l}\right) \circ \cdots \circ M\left(\alpha_{2}\right) \circ M\left(\alpha_{1}\right)$; for each trivial path $e_{a}$, we set $M\left(e_{a}\right)=\mathbb{1}_{M(a)}$. Given two representations $M$ and $N$, a morphism $f: M \rightarrow N$ is given by $k$-linear maps $f(a): M(a) \rightarrow N(a)$ for any vertex $a$, such that $f(b) \circ M(\alpha)=N(\alpha) \circ f(a)$ for any arrow $\alpha: a \rightarrow b$.

Let $M$ be a representation. Recall that the support supp $M$ of $M$ is the full subquiver of $Q$ generated by vertices $a$ with $M(a) \neq 0$. The socle $\operatorname{soc} M$ of $M$ is the subrepresentation of $M$ such that

$$
(\operatorname{soc} M)(a)=\bigcap_{\alpha \in Q_{1}, s(\alpha)=a} \operatorname{Ker} M(\alpha)
$$

for each vertex $a$. The radical $\operatorname{rad} M$ of $M$ is the subrepresentation of $M$ such that

$$
(\operatorname{rad} M)(a)=\sum_{\alpha \in Q_{1}, t(\alpha)=a} \operatorname{Im} M(\alpha)
$$

for each vertex $a$. The top top $M$ of $M$ is the factor representation $M / \operatorname{rad} M$.

We mention the following observation; compare [4, Lemma 1.1].

Lemma 3.1. Let $M$ be a representation.

(1) If $\operatorname{supp} M$ contains no right infinite paths, then $\operatorname{rad} M$ is superfluous in $M$.

(2) If $\operatorname{supp} M$ contains no left infinite paths, then $\operatorname{soc} M$ is essential in $M$.

Proof. (1) Let $N$ be a subrepresentation of $M$ with $N+\operatorname{rad} M=M$. We assume $N(a) \neq M(a)$ for some vertex $a$.

We claim that there exists some $a_{1} \in a^{-}$such that $N\left(a_{1}\right) \neq M\left(a_{1}\right)$. Indeed, otherwise $(\operatorname{rad} M)(a) \subseteq N(a)$ and then $(\operatorname{rad} M+N)(a)=N(a) \neq M(a)$, which is a contradiction.

Set $a_{0}=a$. For every $i \geq 0$, we can find some $a_{i+1} \in a_{i}^{-}$inductively such that $N\left(a_{i+1}\right) \neq M\left(a_{i+1}\right)$. Then we obtain some right infinite path in supp $M$, which is a contradiction. It follows that $N=M$, and then $\operatorname{rad} M$ is superfluous in $M$.

(2) Let $N$ be a nonzero subrepresentation of $M$. Let $a$ be a vertex in $\operatorname{supp} N$. Assume $x$ is a nonzero element in $N(a)$. Since supp $M$ contains no left infinite paths, there exists some finite path $p$ in $\operatorname{supp} M$ with $s(p)=a$ such that $N(p)(x) \neq 0$ and $N(\alpha p)(x)=0$ for any arrow $\alpha$ in $Q$. Therefore $N(p)(x)$ lies in $N \cap \operatorname{soc} M$. It follows that soc $M$ is essential in $M$.

Denote by $\operatorname{Rep}(Q)$ the category of representations of $Q$ over $k$. For every pair of representations $M$ and $N$, denote by $\operatorname{Hom}(M, N)$ the set of morphisms from $M$ to $N$ in $\operatorname{Rep}(Q)$. It is well known that $\operatorname{Rep}(Q)$ is a hereditary abelian category; see [9, Section 8.2].

We associate each representation $M$ of $Q$ with a representation $D M$ of $Q^{\text {op }}$ as follows. For each vertex $a$ in $Q^{\text {op }}$, we let

$$
(D M)(a)=\operatorname{Hom}_{k}(M(a), k) .
$$

For each arrow $\alpha^{\mathrm{op}}: b \rightarrow a$ in $Q^{\mathrm{op}}$, we let

$$
(D M)\left(\alpha^{\mathrm{op}}\right)=\operatorname{Hom}_{k}(M(\alpha), k):(D M)(b) \longrightarrow(D M)(a) .
$$


Given a morphism $f: M \rightarrow N$ in $\operatorname{Rep}(Q)$, we set the morphism $D f: D N \rightarrow D M$ in $\operatorname{Rep}\left(Q^{\text {op }}\right)$ such that

$$
(D f)(a)=\operatorname{Hom}_{k}(f(a), k):(D N)(a) \longrightarrow(D M)(a) .
$$

Then we obtain an exact contravariant functor

$$
D: \operatorname{Rep}(Q) \longrightarrow \operatorname{Rep}\left(Q^{\text {op }}\right) \text {. }
$$

Let $a$ be a vertex in $Q$. We define the representation $P_{a}$ as follows. For each vertex $b$, we let

$$
P_{a}(b)=\bigoplus_{p \in Q(a, b)} k p .
$$

We mention that $P_{a}(b)=0$ if $Q(a, b)=\emptyset$. For each arrow $\alpha: b \rightarrow b^{\prime}$, we let

$$
P_{a}(\alpha): P_{a}(b) \longrightarrow P_{a}\left(b^{\prime}\right), \quad p \mapsto \alpha p,
$$

for any finite path $p \in Q(a, b)$.

Denote by $P_{a}^{\text {op }}$ the corresponding representation of $Q^{\text {op }}$ and let $I_{a}=D P_{a}^{\text {op }}$ in $\operatorname{Rep}(Q)$. We mention that for each vertex $b$, we have that

$$
I_{a}(b)=\operatorname{Hom}_{k}\left(\bigoplus_{p \in Q(b, a)} k p, k\right) .
$$

For each arrow $\alpha: b \rightarrow b^{\prime}$, we have that

$$
I_{a}(\alpha): I_{a}(b) \longrightarrow I_{a}\left(b^{\prime}\right), \quad f \mapsto(p \mapsto f(p \alpha)),
$$

for any $f \in I_{a}(b)$ and any finite path $p \in Q\left(b^{\prime}, a\right)$.

Let $S_{a}$ be the simple representation such that $S_{a}(a)=k e_{a}$ and $S_{a}(b)=0$ for any vertex $b \neq a$. We observe that top $P_{a} \simeq S_{a} \simeq \operatorname{soc} I_{a}$.

The following result seems well known; see [9, Section 3.7]. It implies that $P_{a}$ is a projective representation and $I_{a}$ is an injective representation in $\operatorname{Rep}(Q)$.

Lemma 3.2. Let $M \in \operatorname{Rep}(Q)$ and $a \in Q_{0}$.

(1) The k-linear map

$$
\eta_{M}: \operatorname{Hom}\left(P_{a}, M\right) \longrightarrow M(a),
$$

given by $\eta_{M}(f)=f(a)\left(e_{a}\right)$ for any $f \in \operatorname{Hom}\left(P_{a}, M\right)$, is an isomorphism natural in $M$.

(2) The k-linear map

$$
\zeta_{M}: \operatorname{Hom}\left(M, I_{a}\right) \longrightarrow \operatorname{Hom}_{k}(M(a), k),
$$

given by $\zeta_{M}(f)(x)=f(a)(x)\left(e_{a}\right)$ for any $f \in \operatorname{Hom}\left(M, I_{a}\right)$ and $x \in M(a)$, is an isomorphism natural in $M$.

Proof. (1) Consider the $k$-linear map

$$
\eta_{M}^{\prime}: M(a) \longrightarrow \operatorname{Hom}\left(P_{a}, M\right)
$$

given by $\eta_{M}^{\prime}(x)(b)(p)=M(p)(x)$, for any $x \in M(a)$ and any $b \in Q_{0}$ and $p \in Q(a, b)$. By a direct verification, we have that

$$
\eta_{M}^{\prime} \circ \eta_{M}=\mathbb{1}_{\operatorname{Hom}\left(P_{a}, M\right)} \quad \text { and } \quad \eta_{M} \circ \eta_{M}^{\prime}=\mathbb{1}_{M(a)} .
$$

It follows that $\eta_{M}$ is an isomorphism. The naturality is a direct verification.

(2) Consider the $k$-linear map

$$
\zeta_{M}^{\prime}: \operatorname{Hom}_{k}(M(a), k) \longrightarrow \operatorname{Hom}\left(M, I_{a}\right),
$$

given by $\zeta_{M}^{\prime}(f)(b)(x)(p)=f(M(p)(x))$ for any $f \in \operatorname{Hom}_{k}(M(a), k)$ and any $b \in Q_{0}$, $x \in M(b)$ and $p \in Q(b, a)$. By a direct verification, we have that

$$
\zeta_{M}^{\prime} \circ \zeta_{M}=\mathbb{1}_{\operatorname{Hom}\left(M, I_{a}\right)} \quad \text { and } \quad \zeta_{M} \circ \zeta_{M}^{\prime}=\mathbb{1}_{\operatorname{Hom}_{k}(M(a), k)} .
$$


It follows that $\zeta_{M}$ is an isomorphism. The naturality is a direct verification.

Let $M$ be a representation. Then there exists some epimorphism $\bigoplus_{i \in \Lambda} P_{a_{i}} \rightarrow M$ with $a_{i} \in Q_{0}$. We call $M$ countably generated if $\Lambda$ can be chosen as a countable set; we call $M$ finitely generated if $\Lambda$ can be chosen as a finite set. Dually, there exists some monomorphism $M \rightarrow \prod_{i \in \Lambda} I_{a_{i}}$ with $a_{i} \in Q_{0}$. We call $M$ countably cogenerated if $\Lambda$ can be chosen as a countable set; we call $M$ finitely cogenerated if $\Lambda$ can be chosen as a finite set.

Denote by $\operatorname{proj}(Q)$ the category of finitely generated projective representations and by $\operatorname{inj}(Q)$ the category of finitely cogenerated injective representations.

If $Q$ contains no oriented cycles, we observe by Lemma 3.2 that $\operatorname{End}\left(P_{a}\right) \simeq k \simeq$ $\operatorname{End}\left(I_{a}\right)$ for any vertex $a$. Therefore, both $\operatorname{proj}(Q)$ and $\operatorname{inj}(Q)$ are Krull-Schmidt categories. Every object in $\operatorname{proj}(Q)$ is of the form $\bigoplus_{i=1}^{n} P_{a_{i}}$ with $a_{i} \in Q_{0}$, and every object in $\operatorname{inj}(Q)$ is of the form $\bigoplus_{i=1}^{n} I_{a_{i}}$ with $a_{i} \in Q_{0}$. Moreover, it follows from Azumaya's decomposition theorem that every projective representation in $\operatorname{Rep}(Q)$ is the direct sum of representations of the form $P_{a}$; see [1, Theorem 12.6].

Recall that a representation $M$ is called pointwise finite dimensional if $M(a)$ is finite dimensional for each vertex $a$. We denote by $\operatorname{rep}(Q)$ the category of pointwise finite dimensional representations. The restriction of $D$ gives a duality

$$
D: \operatorname{rep}(Q) \longrightarrow \operatorname{rep}\left(Q^{\mathrm{op}}\right) \text {. }
$$

The following fact is well known.

Lemma 3.3. $\operatorname{rep}(Q)$ is a hereditary abelian subcategory of $\operatorname{Rep}(Q)$, which is closed under extensions.

Proof. One can see that $\operatorname{rep}(Q)$ is an abelian subcategory of $\operatorname{Rep}(Q)$, which is closed under extensions. For any $M$ and $N$ in $\operatorname{rep}(Q)$, we will view $\operatorname{Ext}^{1}(M, N)$ in the sense of Yoneda under the Baer sum. Since $\operatorname{Rep}(Q)$ is hereditary, the functors $\operatorname{Ext}^{1}(M,-)$ and $\operatorname{Ext}^{1}(-, M)$ from $\operatorname{Rep}(Q)$ to $\operatorname{Mod} k$ are right exact. Then so are their restrictions to $\operatorname{rep}(Q)$, since $\operatorname{rep}(Q)$ is closed under extensions. That is to say, $\operatorname{rep}(Q)$ is hereditary.

Given infinitely many objects in $\operatorname{rep}(Q)$, it depends on their supports whether they admit a direct sum in $\operatorname{rep}(Q)$.

Proposition 3.4. Let $M_{i}$ for $i \in \Lambda$ be infinitely many objects in $\operatorname{rep}(Q)$. The following statements are equivalent.

(1) The product of $M_{i}$ for $i \in \Lambda$ exists in $\operatorname{rep}(Q)$.

(2) The coproduct of $M_{i}$ for $i \in \Lambda$ exists in $\operatorname{rep}(Q)$.

(3) For every vertex $a$, there exist only finitely many $i \in \Lambda$ such that $M_{i}(a) \neq 0$.

Proof. $(1) \Rightarrow(3)$. Let $\left(M, f_{i}: M \rightarrow M_{i}\right)$ be the product. Assume there exist infinitely many $i \in \Lambda$ such that $M_{i}(a) \neq 0$, for some vertex $a$. From these elements, we can choose some $i_{1}, i_{2}, \ldots, i_{n}$ such that $n>\operatorname{dim} M(a)$.

Denote by $g_{i}: M_{i} \rightarrow M$ the induced morphism such that $f_{i} \circ g_{i}=\mathbb{1}_{M_{i}}$ and $f_{j} \circ g_{i}=0$ for any $j \neq i$. Consider the morphisms $g: \bigoplus_{j=1}^{n} M_{i_{j}} \rightarrow M$ induced by $g_{i}$, and $f: M \rightarrow \bigoplus_{j=1}^{n} M_{i_{j}}$ induced by $f_{i}$. One can check that $f \circ g=\mathbb{1}_{\bigoplus_{j=1}^{n}} M_{i_{j}}$. In particular, $\operatorname{dim} M(a) \geq \operatorname{dim} \bigoplus_{j=1}^{n} M_{i_{j}}(a) \geq n$, which is a contradiction.

$(3) \Rightarrow(1)$. By the assumption, $\prod_{i \in \Lambda} M_{i}(a)$ is a finite direct product for any vertex $a$. Then the direct product $\prod_{i \in \Lambda} M_{i}$ in $\operatorname{Rep}(Q)$ lies in $\operatorname{rep}(Q)$. It is the product of $M_{i}$ for $i \in \Lambda$ in $\operatorname{rep}(Q)$.

The proof of $(2) \Leftrightarrow(3)$ is similar. 
If the equivalent conditions in the preceding proposition hold, then the product and coproduct are just the direct sum in $\operatorname{Rep}(Q)$, which we denote by $\bigoplus_{i \in \Lambda} M_{i}$. Moreover, $\bigoplus_{i \in \Lambda} M_{i}(a)$ is a finite direct sum for every vertex $a$.

Example 3.5. Let $Q$ be the following quiver

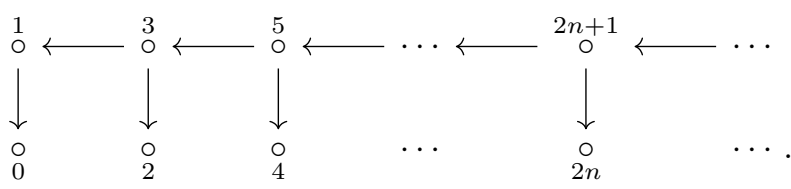

We observe that $P_{2 n} \simeq S_{2 n}$ for $n \geq 0$, and they admit a direct sum $\bigoplus_{n \geq 0} P_{2 n}$ in $\operatorname{rep}(Q)$. Since $P_{2 n+1}(1) \neq 0$ for any $n \geq 0$, then $P_{2 n+1}$ for $n \geq 0$ do not admit a direct sum in $\operatorname{rep}(Q)$ by Proposition 3.4 . Indeed, $\bigoplus_{n \geq 0} P_{2 n+1}$ does not lie in $\operatorname{rep}(Q)$.

The following fact may be known to experts. One can see [11, Theorem 1] for details; compare [2, Main Theorem(a)].

Lemma 3.6. Every $M \in \operatorname{rep}(Q)$ admits a decomposition $M=\bigoplus_{i \in \Lambda} M_{i}$ such that each $M_{i}$ is indecomposable and $\operatorname{End}\left(M_{i}\right)$ is local.

\section{DiRECT Limits AND INVERSE Limits}

In this section, we mention some facts about direct limits and inverse limits.

Let $\left(M_{i}, \psi_{i j}: M_{i} \rightarrow M_{j}\right)$ be a direct system over a directed set $(\Lambda, \leq)$ in $\operatorname{Mod} k$. For each $i \in \Lambda$, we set

$$
M_{i}^{\prime}=\sum_{i \leq j} \operatorname{Ker} \psi_{i j}
$$

We mention the following observations.

Lemma 4.1. For any $i \in \Lambda$ and $x \in M_{i}^{\prime}$, there exists some $l \geq i$ such that $x \in \operatorname{Ker} \psi_{i l}$.

Proof. Assume $x=\sum_{r=1}^{n} x_{r}$ with each $x_{r} \in \operatorname{Ker} \psi_{i j_{r}}$ for some $j_{r} \geq i$. We can choose some $l \geq j_{1}, j_{2}, \ldots, j_{n}$, since $\Lambda$ is directed. We observe that

$$
\psi_{i l}(x)=\sum_{r=1}^{n} \psi_{i l}\left(x_{r}\right)=\sum_{r=1}^{n}\left(\psi_{j_{r} l} \circ \psi_{i j_{r}}\right)\left(x_{r}\right)=0 .
$$

Then the result follows.

Lemma 4.2. For any $i \leq j$, the pre-image of $M_{j}^{\prime}$ under $\psi_{i j}$ is $M_{i}^{\prime}$.

Proof. Let $x \in M_{i}^{\prime}$. Applying Lemma 4.1 for $i$ and $x$, we have some $l \geq i$ such that $x \in \operatorname{Ker} \psi_{i l}$. Choose some $l^{\prime} \geq j, l$. Then

$$
\left(\psi_{j l^{\prime}} \circ \psi_{i j}\right)(x)=\psi_{i l^{\prime}}(x)=\left(\psi_{l l^{\prime}} \circ \psi_{i l}\right)(x)=0 .
$$

Hence $\psi_{i j}(x) \in \operatorname{Ker} \psi_{j l^{\prime}} \subseteq M_{j}^{\prime}$. That is to say, the image of $M_{i}^{\prime}$ under $\psi_{i j}$ is contained in $M_{j}^{\prime}$.

On the other hand, let $x \in M_{i}$ with $\psi_{i j}(x) \in M_{j}^{\prime}$. Applying Lemma 4.1 for $j$ and $\psi_{i j}(x)$, we have some $l \geq j$ such that $\psi_{i j}(x) \in \operatorname{Ker} \psi_{j l}$. Then

$$
\psi_{i l}(x)=\left(\psi_{j l} \circ \psi_{i j}\right)(x)=0 .
$$

Hence $x \in \operatorname{Ker} \psi_{i l} \subseteq M_{i}^{\prime}$. Then the result follows. 
By Lemma 4.2. for any $i \leq j$, we can consider the restriction $\left.\psi_{i j}\right|_{M_{i}^{\prime}}: M_{i}^{\prime} \rightarrow M_{j}^{\prime}$ of $\psi_{i j}$, and the morphism $\overline{\psi_{i j}}: M_{i} / M_{i}^{\prime} \rightarrow M_{j} / M_{j}^{\prime}$ induced by $\psi_{i j}$. Then we obtain direct systems $\left(M_{i}^{\prime},\left.\psi_{i j}\right|_{M_{i}^{\prime}}\right)$ and $\left(M_{i} / M_{i}^{\prime}, \overline{\psi_{i j}}\right)$ over $\Lambda$. Moreover, it follows from Lemma 4.2 that each $\overline{\psi_{i j}}$ is an injection.

The following result shows that the direct system $\left(M_{i} / M_{i}^{\prime}, \overline{\psi_{i j}}\right)$ admits the same direct limit with $\left(M_{i}, \psi_{i j}\right)$.

Proposition 4.3. The canonical map $\phi_{i}: M_{i} / M_{i}^{\prime} \rightarrow \underline{\lim } M_{i} / M_{i}^{\prime}$ is an injection for each $i \in \Lambda$, and $\lim _{\longrightarrow} M_{i} / M_{i}^{\prime} \simeq \lim _{\longrightarrow} M_{i}$.

Proof. For any $i \in \Lambda$ and any $x+M_{i}^{\prime} \in M_{i} / M_{i}^{\prime}$, we mention that $\phi_{i}\left(x+M_{i}^{\prime}\right)=0$ if and only if $\overline{\psi_{i l}}\left(x+M_{i}^{\prime}\right)=0$ for some $l \geq i$; see [17, Lemma 5.30]. We observe by Lemma 4.2 that $\overline{\psi_{i j}}$ is an injection for any $j \geq i$. It follows that $\phi_{i}$ is an injection.

Consider the following exact sequence of direct systems

$$
0 \longrightarrow\left(M_{i}^{\prime}\right) \longrightarrow\left(M_{i}\right) \longrightarrow\left(M_{i} / M_{i}^{\prime}\right) \longrightarrow 0 .
$$

We obtain by [19, Theorem 2.6.15] the exact sequence

$$
0 \longrightarrow \underline{\lim } M_{i}^{\prime} \longrightarrow \lim _{\longrightarrow} M_{i} \longrightarrow \lim _{\longrightarrow} M_{i} / M_{i}^{\prime} \longrightarrow 0
$$

By Lemma 4.1. for any $i \in \Lambda$ and $x \in M_{i}^{\prime}$, there exists some $j \geq i$ such that $x \in \operatorname{Ker} \psi_{i j}$. That is to say $\psi_{i j}(x)=0$. We then obtain $\lim _{\longrightarrow} M_{i}^{\prime}=0$. It follows that $\lim _{\longrightarrow} M_{i} / M_{i}^{\prime} \simeq \lim _{\longrightarrow} M_{i}$.

Based on the above procedure, we can reduce the structure morphisms in a direct system into monomorphisms; see Proposition 5.2 .

Let $\left(M_{i}, \psi_{j i}: M_{j} \rightarrow M_{i}\right)$ be an inverse system over $\Lambda$ in $\operatorname{Mod} k$. It admits the inverse limit $\lim M_{i}$, which is the subspace of $\prod_{i \in \Lambda} M_{i}$ consisting of threads; see [17. Proposition 5.17]. Here, a thread means an element $\left(m_{i}\right) \in \prod_{i \in \Lambda} M_{i}$ such that $\psi_{j i}\left(m_{j}\right)=m_{i}$ for any $i \leq j$.

Recall that $\left(M_{i}, \psi_{j i}\right)$ is said to satisfy the Mittag-Leffler condition, if for each $i \in \Lambda$, there exists some $j \geq i$ such that $\psi_{j i}\left(M_{j}\right)=\psi_{l i}\left(M_{l}\right)$ for any $l \geq j$. We mention that if each $M_{i}$ is finite dimensional, then $\left(M_{i}, \psi_{j i}\right)$ satisfies the MittagLeffler condition naturally.

The following fact is well known; see [19, Proposition 3.5.7].

Lemma 4.4. Assume

$$
0 \longrightarrow\left(U_{i}\right) \longrightarrow\left(V_{i}\right) \longrightarrow\left(W_{i}\right) \longrightarrow 0
$$

is a short exact sequence of inverse systems over $\Lambda$ in $\operatorname{Mod} k$. Then the following induced sequence of inverse limits

$$
0 \longrightarrow \lim _{\longleftarrow} U_{i} \longrightarrow \lim _{\longleftarrow} V_{i} \stackrel{g}{\longrightarrow} \lim _{\longleftarrow} W_{i}
$$

is exact. If moreover $\left(U_{i}\right)$ satisfies the Mittag-Leffler condition and $\Lambda$ is countable, then $g$ is surjective.

The following result is crucial. It will be used to show the projective property of some flat representation in $\operatorname{rep}(Q)$; see Section 6 .

Proposition 4.5. Let $\mathcal{C}$ be a $k$-linear category and let $\Lambda$ be a countable directed set. Assume that $\left(M_{i}, \psi_{i j}: M_{i} \rightarrow M_{j}\right)$ is a direct system over $\Lambda$ in $\mathcal{C}$, with the direct limit $\left(M, \phi_{i}: M_{i} \rightarrow M\right)$. If some morphism $h: M \rightarrow V$ satisfies that each morphism $h \circ \phi_{i}$ factors through $g: U \rightarrow V$ and each $k$-linear space $\operatorname{Hom}_{\mathcal{C}}\left(M_{i}, U\right)$ is finite dimensional, then $h$ factors through $g$. 


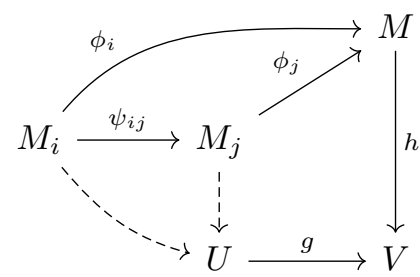

Proof. For any $i \leq j$ and $W \in \mathcal{C}$, we consider the $k$-linear map

$$
\operatorname{Hom}_{\mathcal{C}}\left(\psi_{i j}, W\right): \operatorname{Hom}_{\mathcal{C}}\left(M_{j}, W\right) \longrightarrow \operatorname{Hom}_{\mathcal{C}}\left(M_{i}, W\right), \quad f \mapsto f \circ \psi_{i j},
$$

induced by $\psi_{i j}$. Then $\left(\operatorname{Hom}_{\mathcal{C}}\left(M_{i}, W\right), \operatorname{Hom}_{\mathcal{C}}\left(\psi_{i j}, W\right)\right)$ forms an inverse system in $\operatorname{Mod} k$. We mention the natural isomorphism

$$
\theta_{W}: \operatorname{Hom}_{\mathcal{C}}(M, W) \stackrel{\simeq}{\longrightarrow} \lim _{\leftarrow} \operatorname{Hom}_{\mathcal{C}}\left(M_{i}, W\right), \quad f \mapsto\left(f \circ \phi_{i}\right) .
$$

For each $i \in \Lambda$, we have the $k$-linear map

$$
\operatorname{Hom}_{\mathcal{C}}\left(M_{i}, g\right): \operatorname{Hom}_{\mathcal{C}}\left(M_{i}, U\right) \longrightarrow \operatorname{Hom}_{\mathcal{C}}\left(M_{i}, V\right), \quad f \mapsto g \circ f,
$$

induced by $g$. Express it as the composition of a surjection and the inclusion

$$
\operatorname{Hom}_{\mathcal{C}}\left(M_{i}, g\right): \operatorname{Hom}_{\mathcal{C}}\left(M_{i}, U\right) \stackrel{u_{i}}{\longrightarrow} \operatorname{Im}_{\operatorname{Hom}}\left(M_{i}, g\right) \stackrel{\text { inc }_{i}}{\subseteq} \operatorname{Hom}_{\mathcal{C}}\left(M_{i}, V\right) .
$$

Consider the induced exact sequence

$$
0 \longrightarrow \operatorname{Ker}_{\operatorname{Hom}}\left(M_{i}, g\right) \longrightarrow \operatorname{Hom}_{\mathcal{C}}\left(M_{i}, U\right) \stackrel{u_{i}}{\longrightarrow} \operatorname{Im}_{\operatorname{Hom}_{\mathcal{C}}}\left(M_{i}, g\right) \longrightarrow 0 .
$$

We observe that each $\operatorname{Ker}_{\operatorname{Hom}}\left(M_{i}, g\right)$ is finite dimensional. Then the inverse system $\left(\operatorname{Ker} \operatorname{Hom}_{\mathcal{C}}\left(M_{i}, g\right)\right)$ satisfies the Mittag-Leffler condition. By Lemma 4.4. we obtain the following exact sequence of inverse limits

$$
0 \rightarrow \underset{\lim }{\longleftarrow} \operatorname{Ker}_{\operatorname{Hom}_{\mathcal{C}}}\left(M_{i}, g\right) \rightarrow \underset{\lim }{\longleftarrow} \operatorname{Hom}_{\mathcal{C}}\left(M_{i}, U\right) \stackrel{\overleftarrow{u_{i}}}{\longleftrightarrow} \underset{\lim }{\longleftarrow} \operatorname{Im}_{\operatorname{Hom}}\left(M_{i}, g\right) \rightarrow 0 .
$$

Here, $\overleftarrow{u_{i}}$ is the induced map. Then we obtain the commutative triangle

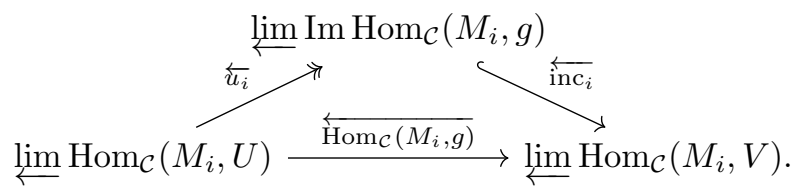

Here, $\overleftarrow{\text { nc }_{i}}$ is an injection by Lemma 4.4 .

For any $i \leq j$, we observe that

$$
\operatorname{Hom}_{\mathcal{C}}\left(\psi_{i j}, V\right)\left(h \circ \phi_{j}\right)=h \circ \phi_{j} \circ \psi_{i j}=h \circ \phi_{i} .
$$

Then $\left(h \circ \phi_{i}\right)$ is an element in $\lim _{\mathcal{C}} \operatorname{Hom}_{\mathcal{C}}\left(M_{i}, V\right)$. By the hypotheses, each $h \circ \phi_{i}$ factors through $g$. In other words, $h \circ \phi_{i}$ lies in $\operatorname{Im}_{\operatorname{Hom}}\left(M_{i}, g\right)$. Then $\left(h \circ \phi_{i}\right)$ is

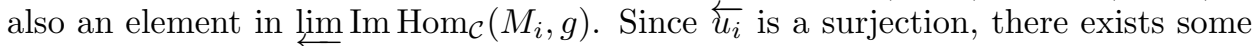
$x \in \lim _{\longleftarrow} \operatorname{Hom}_{\mathcal{C}}\left(M_{i}, U\right)$ such that $\overleftarrow{u_{i}}(x)=\left(h \circ \phi_{i}\right)$. Moreover

$$
\overleftarrow{\operatorname{Hom}_{\mathcal{C}}\left(M_{i}, g\right)}(x)=\overleftarrow{\operatorname{inc}_{i}}\left(\overleftarrow{u_{i}}(x)\right)=\left(\operatorname{inc}_{i}\left(h \circ \phi_{i}\right)\right)=\left(h \circ \phi_{i}\right) .
$$

In other words, $\left(h \circ \phi_{i}\right)$ lies in $\operatorname{Im} \overleftarrow{\operatorname{Hom}_{\mathcal{C}}\left(M_{i}, g\right)}$.

Consider the commutative diagram

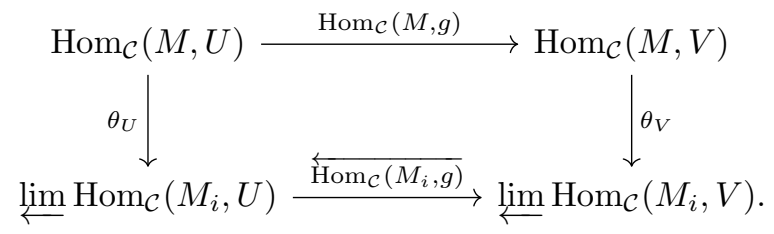


We observe by the isomorphism (4.1) in the case $\theta_{V}$ that $\theta_{V}(h)=\left(h \circ \phi_{i}\right)$. It follows

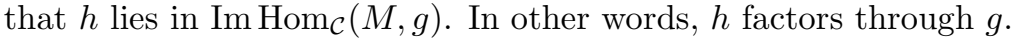

\section{A Class of Flat REPRESEntations}

Let $Q$ be a quiver without oriented cycles. We mention that both $\operatorname{proj}(Q)$ and $\operatorname{inj}(Q)$ are Krull-Schmidt categories.

Recall the Lazard-Govorov Theorem that a module over a unital ring is flat if and only if it is a direct limit of finitely generated free modules; see [10, 13. It is generalized to functor categories as [15, Theorem 3.2]. Especially, a flat representation in $\operatorname{Rep}(Q)$ is a direct limit of finitely generated projective representations. We will introduce a class of flat representations.

Let $[p]$ be an equivalence class of right infinite paths. Given a vertex $a$, we recall that $[p]_{a}$ is the subset of $[p]$ consisting of right infinite paths $q$ with $t(q)=a$.

Inspired by the irreducible representations of Leavitt path algebras introduced in [6. Subsection 3.1] (compare the point modules studied in [18]), we define a representation $X_{[p]}$ as follows. For each vertex $a$, we let

$$
X_{[p]}(a)=\bigoplus_{u \in[p]_{a}} k u \text {. }
$$

We mention that $X_{[p]}(a)=0$ if $[p]_{a}=\emptyset$. For each arrow $\alpha: a \rightarrow b$, we let

$$
X_{[p]}(\alpha): X_{[p]}(a) \longrightarrow X_{[p]}(b), \quad u \mapsto \alpha u,
$$

for any right infinite path $u \in[p]_{a}$.

Assume $p=\alpha_{1} \alpha_{2} \cdots \alpha_{i} \cdots$. We set $a_{i}=t\left(\alpha_{i+1}\right)$ for each $i \geq 0$. Recall that $P_{a}$ is the corresponding projective representation for any vertex $a$. For each $j>i$, the finite path $\alpha_{i+1} \alpha_{i+2} \cdots \alpha_{j}$ induces a morphism $\psi_{i j}: P_{a_{i}} \rightarrow P_{a_{j}}$, such that

$$
\psi_{i j}(b): P_{a_{i}}(b) \longrightarrow P_{a_{j}}(b), \quad u \mapsto u \alpha_{i+1} \alpha_{i+2} \cdots \alpha_{j},
$$

for any vertex $b$ and any finite path $u \in Q\left(a_{i}, b\right)$. Set $\psi_{i i}=\mathbb{1}_{P_{a_{i}}}$. We observe that $\left(P_{a_{i}}, \psi_{i j}\right)$ is a direct system over $(\mathbb{N}, \leq)$.

For each $i \geq 0$, the right infinite path $\alpha_{i+1} \alpha_{i+2} \cdots \alpha_{j} \cdots$ induces a morphism $\phi_{i}: P_{a_{i}} \rightarrow X_{[p]}$, such that

$$
\phi_{i}(b): P_{a_{i}}(b) \longrightarrow X_{[p]}(b), \quad u \mapsto u \alpha_{i+1} \alpha_{i+2} \cdots \alpha_{j} \cdots,
$$

for any vertex $b$ and any finite path $u \in Q\left(a_{i}, b\right)$.

The following result shows that $X_{[p]}$ is a flat representation in $\operatorname{Rep}(Q)$.

Lemma 5.1. $\left(X_{[p]}, \phi_{i}\right)$ is the direct limit of $\left(P_{a_{i}}, \psi_{i j}\right)$ in $\operatorname{Rep}(Q)$.

Proof. We observe that $\phi_{j} \circ \psi_{i j}=\phi_{i}$ for any $i \leq j$. Since $Q$ contains no oriented cycles, the right infinite path $\alpha_{i+1} \alpha_{i+2} \cdots \alpha_{j} \cdots$ is not cyclic. Lemma 2.1 implies that each $\phi_{i}(b)$ is an injection. Then $\operatorname{Im} \phi_{i} \simeq P_{a_{i}}$. Since $\bigcup_{i \geq 0} \operatorname{Im} \phi_{i}=X_{[p]}$, then the result follows.

We observe that the direct system $\left(P_{a_{i}}, \psi_{i j}\right)$ depends on the choice of representative $p$, but the direct limit $X_{[p]}$ does not.

We mention that $X_{[p]} \not P_{a}$ for any vertex $a$. Indeed, we observe that $\operatorname{rad} X_{[p]}=$ $X_{[p]}$ and hence top $X_{[p]}=0$. Then the result follows since top $P_{a} \simeq S_{a}$.

One can see from Lemma 5.1 that the flat representation $X_{[p]}$ is the direct limit of finitely generated projective subrepresentations. The following result shows that this property holds for general flat representations in $\operatorname{Rep}(Q)$. It strengthens the Lazard-Govorov Theorem in the special case $\operatorname{Rep}(Q)$.

Proposition 5.2. A flat representation in $\operatorname{Rep}(Q)$ is a direct limit of finitely generated projective subrepresentations. 
Proof. Assume $M$ is the direct limit of a direct system $\left(M_{i}, \psi_{i j}: M_{i} \rightarrow M_{j}\right)$ over a directed set $\Lambda$ in $\operatorname{Rep}(Q)$ with each $M_{i} \in \operatorname{proj}(Q)$. We set $M_{i}^{\prime}=\sum_{i<j} \operatorname{Ker} \psi_{i j}$ for each $i \in \Lambda$. By Lemma 4.2, we can let $\overline{\psi_{i j}}: M_{i} / M_{i}^{\prime} \rightarrow M_{j} / M_{j}^{\prime}$ be the morphism induced by $\psi_{i j}$, and then $\left(M_{i} / M_{i}^{\prime}, \overline{\psi_{i j}}\right)$ forms a direct system. By Proposition 4.3 , we have that $M \simeq \lim _{i} M_{i} / M_{i}^{\prime}$ and each canonical morphism $M_{i} / M_{i}^{\prime} \rightarrow M$ is an injection. It remains to show that $M_{i} / M_{i}^{\prime} \in \operatorname{proj}(Q)$ for any $i \in \Lambda$.

Let $i \in \Lambda$. We observe that $\operatorname{Im} \psi_{i j}$ is projective for any $j \geq i$, since $M_{j}$ is projective and $\operatorname{Rep}(Q)$ is hereditary. We obtain a split exact sequence

$$
0 \longrightarrow \operatorname{Ker} \psi_{i j} \stackrel{u_{j}}{\longrightarrow} M_{i} \longrightarrow \operatorname{Im} \psi_{i j} \longrightarrow 0 .
$$

Here $u_{j}$ is the inclusion. Then $\operatorname{Im} \psi_{i j}$ and $\operatorname{Ker} \psi_{i j}$ are direct summands of $M_{i}$, and hence lie in $\operatorname{proj}(Q)$. We mention that there exists some morphism $v_{j}: M_{i} \rightarrow$ $\operatorname{Ker} \psi_{i j}$ such that $v_{j} \circ u_{j}=\mathbb{1}_{\operatorname{Ker} \psi_{i j}}$.

For any $j^{\prime} \geq j \geq i$, we can consider the inclusions $u_{j^{\prime}}$ : Ker $\psi_{i j^{\prime}} \rightarrow M_{i}$ and $w: \operatorname{Ker} \psi_{i j} \rightarrow \operatorname{Ker} \psi_{i j^{\prime}}$. We observe that $u_{j^{\prime}} \circ w=u_{j}$ and hence $\left(v_{j} \circ u_{j^{\prime}}\right) \circ w=$ $v_{j} \circ u_{j}=\mathbb{1}_{\text {Ker } \psi_{i j}}$. It follows that Ker $\psi_{i j}$ is a direct summand of Ker $\psi_{i j^{\prime}}$. We may assume $M_{i} \simeq \bigoplus_{r=1}^{n} P_{a_{r}}$ for some vertices $a_{1}, a_{2}, \ldots, a_{n}$. Then the number of indecomposable direct summands of Ker $\psi_{i j}$ for a certain decomposition is less than or equal to the one of $\operatorname{Ker} \psi_{i j^{\prime}}$, and they are both less than or equal to $n$. It follows that there exists some $l \geq i$ such that $\operatorname{Ker} \psi_{i l}=\operatorname{Ker} \psi_{i j}$ for any $j \geq l$.

For any $j \geq i$, we can choose some $j^{\prime} \geq j, l$. We have that $\operatorname{Ker} \psi_{i j} \subseteq \operatorname{Ker} \psi_{i j^{\prime}}=$ $\operatorname{Ker} \psi_{i l}$. We then obtain $M_{i}^{\prime}=\operatorname{Ker} \psi_{i l}$. It follows from the exact sequence (5.1) in the case $\psi_{i l}$ that $M_{i} / M_{i}^{\prime}=M_{i} / \operatorname{Ker} \psi_{i l} \simeq \operatorname{Im} \psi_{i l}$ and hence $M_{i} / M_{i}^{\prime} \in \operatorname{proj}(Q)$.

We study morphisms between representations of the form $X_{[p]}$.

Lemma 5.3. Let $[p]$ and $[q]$ be equivalence classes of right infinite paths. Assume $g: X_{[p]} \rightarrow X_{[q]}$ is a nonzero morphism. Then $[p]=[q]$ and $g=r \mathbb{1}_{X_{[p]}}$ for some nonzero $r \in k$.

Proof. Assume $p=\alpha_{1} \alpha_{2} \cdots \alpha_{i} \cdots$. For each $i \geq 0$, we set $a_{i}=t\left(\alpha_{i+1}\right)$. We observe that for any vertex $a$ and any right infinite path $p^{\prime}$ in $[p]_{a}$, there exists some $i \geq 0$ and some finite path $w \in Q\left(a_{i}, a\right)$ such that $p^{\prime}=w \alpha_{i+1} \alpha_{i+2} \cdots \alpha_{l} \cdots$. Consider the commutative diagram

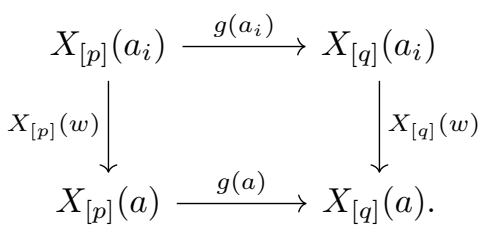

We observe that $p^{\prime}=X_{[p]}(w)\left(\alpha_{i+1} \alpha_{i+2} \cdots \alpha_{l} \cdots\right)$. It follows that

$$
\begin{aligned}
g(a)\left(p^{\prime}\right) & =\left(g(a) \circ X_{[p]}(w)\right)\left(\alpha_{i+1} \alpha_{i+2} \cdots \alpha_{l} \cdots\right) \\
& =\left(X_{[q]}(w) \circ g\left(a_{i}\right)\right)\left(\alpha_{i+1} \alpha_{i+2} \cdots \alpha_{l} \cdots\right) .
\end{aligned}
$$

We claim that there exists some $i \geq 0$ such that $g\left(a_{i}\right)\left(\alpha_{i+1} \alpha_{i+2} \cdots \alpha_{l} \cdots\right) \neq 0$. Otherwise, for any vertex $a$ and any right infinite path $p^{\prime}$ in $[p]_{a}$, we have $g(a)\left(p^{\prime}\right)=$ 0 by (5.2). It follows that $g=0$, which is a contradiction.

Hence, without loss of generality, we may assume $g\left(a_{0}\right)(p) \neq 0$. Then for any $i>0$, we observe by (5.2) in the case $a=a_{0}, p^{\prime}=p$ and $w=\alpha_{1} \alpha_{2} \cdots \alpha_{i}$ that

$$
0 \neq g\left(a_{0}\right)(p)=\left(X_{[q]}\left(\alpha_{1} \alpha_{2} \cdots \alpha_{i}\right) \circ g\left(a_{i}\right)\right)\left(\alpha_{i+1} \alpha_{i+2} \cdots \alpha_{l} \cdots\right) .
$$

In particular, we have $g\left(a_{i}\right)\left(\alpha_{i+1} \alpha_{i+2} \cdots \alpha_{l} \cdots\right) \neq 0$. 
For each $i \geq 0$, we assume

$$
g\left(a_{i}\right)\left(\alpha_{i+1} \alpha_{i+2} \cdots \alpha_{l} \cdots\right)=\sum_{j=1}^{n_{i}} r_{i j} u_{i j}
$$

where $n_{i} \geq 1,0 \neq r_{i j} \in k$, and $u_{i j} \in[q]_{a_{i}}$.

We observe that

$$
\begin{aligned}
\sum_{j=1}^{n_{i}} r_{i j} u_{i j} & =g\left(a_{i}\right)\left(\alpha_{i+1} \alpha_{i+2} \cdots \alpha_{l} \cdots\right) \\
& =\left(X_{[q]}\left(\alpha_{i+1}\right) \circ g\left(a_{i+1}\right)\right)\left(\alpha_{i+2} \alpha_{i+3} \cdots \alpha_{l} \cdots\right) \\
& =X_{[q]}\left(\alpha_{i+1}\right)\left(\sum_{j=1}^{n_{i+1}} r_{i+1, j} u_{i+1, j}\right) \\
& =\sum_{j=1}^{n_{i+1}} r_{i+1, j} \alpha_{i+1} u_{i+1, j} .
\end{aligned}
$$

Here, the first equality is (5.3). The second equality is (5.2) in the case $a=a_{i}$, $p^{\prime}=\alpha_{i+1} \alpha_{i+2} \cdots \alpha_{l} \cdots$ and $w=\alpha_{i+1}$. The third equality holds by (5.3) in the case $i+1$. The fourth equality holds by the definition of $X_{[q]}\left(\alpha_{i+1}\right)$.

Therefore, we have that $n_{i}=n_{i+1}$. For each $1 \leq j \leq n_{i}$, there exists some $1 \leq j^{\prime} \leq n_{i+1}$ such that $u_{i j}=\alpha_{i+1} u_{i+1, j^{\prime}}$ and $r_{i j}=r_{i+1, j^{\prime}}$.

By induction, we have that

$$
u_{0 j}=\alpha_{1} \alpha_{2} \cdots \alpha_{l} \cdots=p
$$

for each $1 \leq j \leq n_{0}$ and hence $n_{0}=1$. It follows that $[p]=[q]$ since $u_{0 j} \in[q]$.

Let $r=r_{01}$. Then for each $i \geq 0$, we have

$$
g\left(a_{i}\right)\left(\alpha_{i+1} \alpha_{i+2} \cdots \alpha_{l} \cdots\right)=r \alpha_{i+1} \alpha_{i+2} \cdots \alpha_{l} \cdots .
$$

For any vertex $a$ and any right infinite path $p^{\prime}$ in $[p]_{a}$, we may assume $p^{\prime}=$ $w \alpha_{i+1} \alpha_{i+2} \cdots \alpha_{l} \cdots$ for some $i \geq 0$ and some finite path $w \in Q\left(a_{i}, a\right)$. By (5.2) and (5.4), we have that

$$
\begin{aligned}
g(a)\left(p^{\prime}\right) & =\left(X_{[p]}(w) \circ g\left(a_{i}\right)\right)\left(\alpha_{i+1} \alpha_{i+2} \cdots \alpha_{l} \cdots\right) \\
& =X_{[p]}(w)\left(r \alpha_{i+1} \alpha_{i+2} \cdots \alpha_{l} \cdots\right) \\
& =r p^{\prime} .
\end{aligned}
$$

It follows that $g=r \mathbb{1}_{X_{[p]}}$.

We mention that Lemma 5.3 still holds even if $Q$ contains oriented cycles. The following result is a direct consequence of Lemma 5.3. It implies that the representations of the form $X_{[p]}$ are indecomposable and pairwise non-isomorphic.

Proposition 5.4. Let $[p]$ and $[q]$ be equivalence classes of right infinite paths.

(1) $X_{[p]} \simeq X_{[q]}$ if and only if $[p]=[q]$.

(2) $\operatorname{End}\left(X_{[p]}\right) \simeq k$.

We mention the following characterization for $X_{[p]}$ lying in $\operatorname{rep}(Q)$.

Lemma 5.5. Let $[p]$ be an equivalence class of right infinite paths. Then $X_{[p]}$ lies in $\operatorname{rep}(Q)$ if and only if the convex hull of any right infinite path in $[p]$ is uniformly interval finite.

Proof. We observe that $X_{[p]}$ lies in $\operatorname{rep}(Q)$ if and only if $[p]_{a}$ is finite for any $a \in Q_{0}$. Since $Q$ contains no oriented cycles, every right infinite path is irrational. Then the result follows from the equivalence between Proposition 2.5(1) and Proposition $2.5(3)$. 
We mention that the convex hull of any right infinite path in $[p]$ is a convex subquiver of $\operatorname{supp} X_{[p]}$. But $X_{[p]} \in \operatorname{rep}(Q)$ does not imply that $\operatorname{supp} X_{[p]}$ is uniformly interval finite; see the following example.

Example 5.6. Let $Q$ be the following quiver

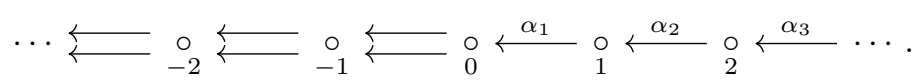

Let $p=\alpha_{1} \alpha_{2} \cdots \alpha_{i} \cdots$. Then $\operatorname{supp} X_{[p]}=Q$. We observe that $\left|[p]_{i}\right|=1$ for each $i \geq 0$ and $|Q(0, i)|=\left|[p]_{i}\right|=2^{-i}$ for each $i<0$. It follows that $X_{[p]} \in \operatorname{rep}(Q)$. But $\operatorname{supp} X_{[p]}$ is not uniformly interval finite.

Let $[p]$ be an equivalence class of left infinite paths. Then $\left[p^{\mathrm{op}}\right]$ is the equivalence class of right infinite paths in $Q^{\mathrm{op}}$. Assume $p=\cdots \alpha_{i} \cdots \alpha_{2} \alpha_{1}$. Set $a_{i}=s\left(\alpha_{i+1}\right)$ for each $i \geq 0$. Then $p^{\mathrm{op}}=\alpha_{1}^{\mathrm{op}} \alpha_{2}^{\mathrm{op}} \cdots \alpha_{i}^{\mathrm{op}} \cdots$ and $a_{i}=t\left(\alpha_{i+1}^{\mathrm{op}}\right)$ for each $i \geq 0$. We have a direct system $\left(P_{a_{i}}^{\mathrm{op}}\right)$ over $\mathbb{N}$ with the direct limit $X_{\left[p^{\mathrm{op}}\right]}$ in $\operatorname{Rep}\left(Q^{\mathrm{op}}\right)$. Applying the contravariant functor $D: \operatorname{Rep}\left(Q^{\mathrm{op}}\right) \rightarrow \operatorname{Rep}(Q)$, we obtain an inverse system $\left(I_{a_{i}}\right)$ over $\mathbb{N}$ in $\operatorname{Rep}(Q)$. Let $Y_{[p]}=D X_{\left[p^{\mathrm{op}}\right]}$ in $\operatorname{Rep}(Q)$.

We observe that $D$ sends direct limits to inverse limits. Then we obtain the following result, which is dual to Lemma 5.1 .

Lemma 5.7. $Y_{[p]}$ is the inverse limit of $\left(I_{a_{i}}\right)$ in $\operatorname{Rep}(Q)$.

We mention that $Y_{[p]} \nsucceq I_{a}$ for any vertex $a$. Indeed, we observe that $\operatorname{soc} Y_{[p]}=0$. Then the result follows since $\operatorname{soc} I_{a} \simeq S_{a}$.

\section{The Classification THEOREM}

Let $Q$ be an interval finite quiver. Then $\operatorname{proj}(Q)$ and $\operatorname{inj}(Q)$ are both subcategories of $\operatorname{rep}(Q)$. We refer to [4] for more details about $\operatorname{rep}(Q)$.

Lemma 6.1. Let $M \in \operatorname{rep}(Q)$ and let $\Lambda$ be a countable directed set. Assume that $M$ is a direct limit of objects $M_{i} \in \operatorname{proj}(Q)$ over $\Lambda$ in $\operatorname{rep}(Q)$. Then $M$ is projective in $\operatorname{rep}(Q)$.

Proof. Assume that $\phi_{i}: M_{i} \rightarrow M$ is the canonical morphism for each $i \in \Lambda$. Let $g: X \rightarrow Y$ be an epimorphism and let $f: M \rightarrow Y$ be a morphism in $\operatorname{rep}(Q)$. We have that each $f \circ \phi_{i}$ factors through $g$ since $M_{i}$ is projective. We observe that $\operatorname{Hom}\left(M_{i}, X\right)$ is finite dimensional. By Proposition 4.5, we have that $f$ factors through $g$. It follows that $M$ is projective in $\operatorname{rep}(Q)$.

Then we can show that $X_{[p]}$ is projective in $\operatorname{rep}(Q)$ if it lies in $\operatorname{rep}(Q)$.

Proposition 6.2. Let $[p]$ be an equivalence class of right infinite paths such that the convex hull of any right infinite path in $[p]$ is uniformly interval finite. Then $X_{[p]}$ is an indecomposable projective object in $\operatorname{rep}(Q)$.

Proof. By Lemma 5.5] and Propositions [5.4(2), we have that $X_{[p]}$ is an indecomposable object in $\operatorname{rep}(Q)$. By Lemma 5.1, $X_{[p]}$ is a direct limit of objects lying in $\operatorname{proj}(Q)$ over $\mathbb{N}$ in $\operatorname{Rep}(Q)$. Then the result follows from Lemma 6.1]

The following result shows that the convex hull of any right infinite path in $\operatorname{supp} M$ is uniformly interval finite, if $M$ is a projective object in $\operatorname{rep}(Q)$.

Lemma 6.3. Let $M$ be a representation in $\operatorname{rep}(Q)$. Assume that $\operatorname{supp} M$ contains a right infinite path $p$ whose convex hull is not uniformly interval finite. Then $M$ is not projective in $\operatorname{rep}(Q)$. 
Proof. Assume $p=\alpha_{1} \alpha_{2} \cdots \alpha_{i} \cdots$. For each $i \geq 0$, we set $a_{i}=t\left(\alpha_{i+1}\right)$. Assume that $M$ is projective in $\operatorname{rep}(Q)$.

We observe that $\operatorname{Hom}\left(P_{a_{i}}, M\right) \simeq M\left(a_{i}\right)$ and hence it is nonzero. Let $f_{i}: P_{a_{i}} \rightarrow$ $M$ be a nonzero morphism. Since $M$ is projective in $\operatorname{rep}(Q)$ which is hereditary, we have that $f_{i}$ is an injection. Then $\operatorname{dim} M\left(a_{0}\right) \geq \operatorname{dim} P_{a_{i}}\left(a_{0}\right)=\left|Q\left(a_{i}, a_{0}\right)\right|$.

Since $Q$ contains no oriented cycles, every right infinite path is irrational. Then the equivalence between Proposition 2.5(1) and Proposition 2.5)(2) implies that $\left\{\left|Q\left(a_{i}, a_{0}\right)\right| \mid i \geq 0\right\}$ is unbounded. It follows that $M\left(a_{0}\right)$ is not finite dimensional, which is a contradiction. Then the result follows.

We mention the following observations about projective objects in $\operatorname{rep}(Q)$.

Lemma 6.4. Let $M$ be a projective object in $\operatorname{rep}(Q)$. Then $M(\alpha)$ is an injection for any arrow $\alpha: a \rightarrow b$.

Proof. We observe that there exists some morphism $f: P_{a}^{\oplus \operatorname{dim} M(a)} \rightarrow M$ such that $\operatorname{Im} f(a)=M(a)$. Since $\operatorname{rep}(Q)$ is hereditary and $M$ is projective, then $f$ is an injection. We observe that $P_{a}(\alpha)$ is an injection. Then the result follows.

For any vertex $a$ and $x \in M(a)$, we denote by $M_{x}$ the subrepresentation of $M$ generated by $x$.

Lemma 6.5. Let $M$ be a projective object in $\operatorname{rep}(Q)$. Assume $a_{1}, a_{2}, \ldots, a_{n}$ are vertices in $\operatorname{supp} M$, and $x_{i} \in M\left(a_{i}\right)$ is nonzero for each $1 \leq i \leq n$ such that $x_{i} \notin \sum_{j \neq i} M_{x_{j}}\left(a_{i}\right)$. Then $\sum_{i=1}^{n} M_{x_{i}}$ is an internal direct sum.

Proof. We mention that $M_{x_{i}} \simeq P_{a_{i}}$ for each $1 \leq i \leq n$. Consider the canonical epimorphism

$$
g: \bigoplus_{i=1}^{n} M_{x_{i}} \longrightarrow \sum_{i=1}^{n} M_{x_{i}} .
$$

Here, the direct sum means external direct sum. We observe that $\sum_{i=1}^{n} M_{x_{i}}$ is projective in $\operatorname{rep}(Q)$, since $\operatorname{rep}(Q)$ is hereditary. Then $g$ is a split epimorphism.

For each $1 \leq i \leq n$, we have that $\left(\operatorname{rad} M_{x_{i}}\right)\left(a_{i}\right)=0$. Then $\left(\operatorname{rad} \sum_{j=1}^{n} M_{x_{j}}\right)\left(a_{i}\right)$ is contained in $\sum_{j \neq i} M_{x_{j}}\left(a_{i}\right)$. By the assumption, we have that $x_{i}$ does not lie in $\left(\operatorname{rad} \sum_{j=1}^{n} M_{x_{j}}\right)\left(a_{i}\right)$. Then $\bigoplus_{i=1}^{n} S_{a_{i}}$ is a direct summand of $\operatorname{top}\left(\sum_{i=1}^{n} M_{x_{i}}\right)$. We observe by the split epimorphism $g$ that $\operatorname{top}\left(\sum_{i=1}^{n} M_{x_{i}}\right)$ is a direct summand of $\operatorname{top}\left(\bigoplus_{i=1}^{n} M_{x_{i}}\right)$, which is isomorphic to $\bigoplus_{i=1}^{n} S_{a_{i}}$. It follows that

$$
\operatorname{top}\left(\sum_{i=1}^{n} M_{x_{i}}\right) \simeq \bigoplus_{i=1}^{n} S_{a_{i}} \simeq \operatorname{top}\left(\bigoplus_{i=1}^{n} M_{x_{i}}\right) .
$$

Since both $\bigoplus_{i=1}^{n} M_{x_{i}}$ and $\sum_{i=1}^{n} M_{x_{i}}$ lie in $\operatorname{proj}(Q)$, then $g$ is an isomorphism. Then the result follows.

Proposition 6.6. Let $M$ be an indecomposable projective object in $\operatorname{rep}(Q)$. Assume that $\operatorname{supp} M$ contains a right infinite path $p$. Then the convex hull of any right infinite path in $[p]$ is uniformly interval finite and $M \simeq X_{[p]}$.

Proof. We observe by Lemma 6.4 that each right infinite path in $[p]$ is completely contained in supp $M$. Then the convex hull of any right infinite path in $[p]$ is uniformly interval finite by Lemma 6.3. It follows from Proposition 6.2 that $X_{[p]}$ is an indecomposable projective object in $\operatorname{rep}(Q)$.

Assume $p=\alpha_{1} \alpha_{2} \cdots \alpha_{i} \cdots$. For each $i \geq 0$, we set $a_{i}=t\left(\alpha_{i+1}\right)$. We have that $\operatorname{dim} M\left(a_{i}\right) \geq \operatorname{dim} M\left(a_{i+1}\right)$ since $M\left(\alpha_{i+1}\right)$ is an injection. Then $\operatorname{dim} M\left(a_{i}\right)$ will be stable for $i$ large enough. We may assume $\operatorname{dim} M\left(a_{i}\right)=\operatorname{dim} M\left(a_{0}\right)$ for each $i \geq 0$. Then $M\left(\alpha_{i}\right)$ is a bijection for each $i \geq 0$. 
Let $\Omega_{0}=\left\{a_{i} \mid i \geq 0\right\}$. We denote by $X_{0}$ the subrepresentation of $M$ generated by $\bigcup_{a \in \Omega_{0}} M(a)$. We observe that

$$
X_{0} \simeq X_{[p]}^{\oplus \operatorname{dim} M\left(a_{0}\right)}
$$

Let $\Omega_{1}=\Omega_{0} \cup Q_{0} \backslash \operatorname{supp} X_{0}$. We denote by $X_{1}$ the subrepresentation of $M$ generated by $\bigcup_{a \in \Omega_{1} \backslash \Omega_{0}} M(a)$. We observe that $X_{0}(a)+X_{1}(a)=M(a)$ for each $a \in \Omega_{1}$.

We claim that $X_{0} \cap X_{1}=0$. Indeed, let $x \in X_{0}(a) \cap X_{1}(a)$ for some vertex $a$. Then there exists pairwise different vertices $b_{1}, b_{2}, \ldots, b_{n}$ in $\Omega_{1} \backslash \Omega_{0}$ and $y_{i} \in X_{1}\left(b_{i}\right)$ for each $1 \leq i \leq n$ such that $x \in \sum_{i=1}^{n} M_{y_{i}}(a)$. We may assume $y_{i} \notin \sum_{j \neq i} M_{y_{j}}\left(b_{i}\right)$ for any $1 \leq i \leq n$. We observe that there exist some $j \geq 0$ such that there does not exist a finite path $u$ with $t(u)=a_{j}$ and $s(u)=b_{i}$ for any $1 \leq i \leq n$, since $Q$ is interval finite. We can choose $y_{n+1} \in M\left(a_{j}\right)$ such that $x \in M_{y_{n+1}}(a)$. We observe that $y_{i} \notin \sum_{j \neq i} M_{y_{j}}\left(b_{i}\right)$ for any $1 \leq i \leq n+1$. By Lemma 6.5, we have that $x=0$. It follows that $X_{0} \cap X_{1}=0$.

Let $\Omega_{2}=\left\{a \in Q_{0} \mid a^{-} \subseteq \Omega_{1}\right\}$. For each $a \in \Omega_{2} \backslash \Omega_{1}$, we let $U_{a}$ be a complement to $X_{0}(a) \oplus X_{1}(a)$ in $M(a)$. Let $X_{2}$ be the subrepresentation of $M$ generated by $\bigcup_{a \in \Omega_{2} \backslash \Omega_{1}} U_{a}$. We observe that $X_{0}(a) \oplus X_{1}(a) \oplus X_{2}(a)=M(a)$ for each $a \in \Omega_{2}$.

We claim that $\left(X_{0} \oplus X_{1}\right) \cap X_{2}=0$. Indeed, let $x \in\left(X_{0} \oplus X_{1}\right)(a) \cap X_{2}(a)$ for some vertex $a$. Then there exist pairwise different vertices $b_{1}, b_{2}, \ldots, b_{n} \in \Omega_{2} \backslash \Omega_{1}$ and $y_{i} \in X_{2}\left(b_{i}\right)$ for each $1 \leq i \leq n$ such that $x \in \sum_{i=1}^{n} M_{y_{i}}(a)$. We may assume $y_{i} \notin \sum_{1 \leq j \leq n, j \neq i} M_{y_{j}}\left(b_{i}\right)$ for any $1 \leq i \leq n$. Similarly, there exist pairwise different vertices $b_{n+1}, b_{n+2}, \ldots, b_{n+m} \in \Omega_{1}$ and $y_{i} \in\left(X_{0} \oplus X_{1}\right)\left(b_{i}\right)$ for each $n+1 \leq i \leq n+m$ such that $x \in \sum_{i=n+1}^{n+m} M_{y_{i}}(a)$. We may assume $y_{i} \notin \sum_{n+1 \leq j \leq n+m, j \neq i} M_{y_{j}}\left(b_{i}\right)$ for any $n+1 \leq i \leq n+m$. We observe that $y_{i} \notin \sum_{j \neq i} M_{y_{j}}\left(b_{i}\right)$ for any $1 \leq i \leq n+m$, since $\sum_{i=0}^{2} X_{i}(a)$ is an internal direct sum for each $a \in \Omega_{2}$. By Lemma 6.5, we have that $x=0$. It follows that $\left(X_{0} \oplus X_{1}\right) \cap X_{2}=0$.

By induction, for each $n \geq 0$, we have $\Omega_{n}$ and $X_{n}$ such that $\sum_{i=1}^{n} X_{i}$ is an internal direct sum and $\left(\bigoplus_{i=0}^{n} X_{i}\right)(a)=M(a)$ for each vertex $a \in \Omega_{n}$.

We observe that any vertex in $\operatorname{supp} M$ lies in $\Omega_{i}$ for some $i \geq 0$. It follows that $M=\bigoplus_{i=0}^{\infty} X_{i}$. Since $M$ is indecomposable, we have that $M=X_{0} \simeq X_{[p]}$.

We then obtain the following classification theorem.

Theorem 6.7. Let $Q$ be an interval finite quiver. Assume that $P$ is an indecomposable projective object in $\operatorname{rep}(Q)$. Then either $P \simeq P_{a}$ for some vertex $a$, or $P \simeq X_{[p]}$ for some equivalence class $[p]$ of right infinite paths, where the convex hull of any right infinite path in $[p]$ is uniformly interval finite.

Proof. If supp $P$ contains a right infinite path, the result follows from Proposition 6.6. Now, we assume that $\operatorname{supp} P$ contains no right infinite paths. Then the canonical epimorphism $P \rightarrow$ top $P$ is a projective cover by Lemma 3.1(1). In particular, top $P$ is nonzero. Let $S$ be a simple factor representation of top $P$. We have an epimorphism $f: P \rightarrow S$. Assume that $S(a) \neq 0$ for some vertex $a$. We have a projective cover $g: P_{a} \rightarrow S$. It follows that $P_{a}$ is a direct summand of $P$. Since $P$ is indecomposable, we have that $P \simeq P_{a}$.

We mention that an indecomposable projective object in $\operatorname{rep}(Q)$ of the form $X_{[p]}$ is not projective in $\operatorname{Rep}(Q)$. Indeed, if $p=\alpha_{1} \alpha_{2} \cdots \alpha_{i} \cdots$ and $a_{i}=t\left(\alpha_{i+1}\right)$ for any $i \geq 0$, then the canonical epimorphism $\bigoplus_{i \geq 0} P_{a_{i}} \rightarrow X_{[p]}$ is not a retraction.

By the duality $D: \operatorname{rep}\left(Q^{\mathrm{op}}\right) \rightarrow \operatorname{rep}(Q)$, we can also classify the injective objects in $\operatorname{rep}(Q)$; see the following theorem. One can also obtain the classification of injective objects in $\operatorname{rep}(Q)$ analogous to the above process based on Lemmas $\underline{3.2}(2)$ and 5.7 
Theorem 6.8. Let $Q$ be an interval finite quiver. Assume that $I$ is an indecomposable injective object in $\operatorname{rep}(Q)$. Then either $I \simeq I_{a}$ for some vertex a, or $I \simeq Y_{[p]}$ for some equivalence class $[p]$ of left infinite paths, where the convex hull of any left infinite path in $[p]$ is uniformly interval finite.

We characterize the indecomposable projective objects and indecomposable injective objects in $\operatorname{rep}(Q)$ for the following infinite quiver of $A_{\infty}^{\infty}$ type.

Example 6.9. Let $Q$ be the following quiver of $A_{\infty}^{\infty}$ type

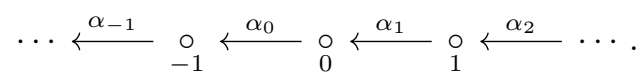

For every vertex $i$, we denote by $P_{i}$ the corresponding indecomposable projective representation, and by $I_{i}$ the corresponding indecomposable injective representation.

Set $p=\alpha_{1} \alpha_{2} \cdots \alpha_{i} \cdots$. Then $X_{[p]}$ is isomorphic to the representation

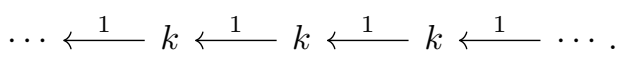

By Proposition 6.2, we have that $X_{[p]}$ is an indecomposable projective object in $\operatorname{rep}(Q)$. By Theorem 6.7, we have that

$$
\left\{X_{[p]}\right\} \cup\left\{P_{i} \mid i \in Q_{0}\right\}
$$

is a complete set of indecomposable projective objects in $\operatorname{rep}(Q)$.

Set $q=\cdots \alpha_{j} \cdots \alpha_{-2} \alpha_{-1}$. Then $Y_{[q]} \simeq X_{[p]}$. By Theorem 6.8, we have that

$$
\left\{Y_{[q]}\right\} \cup\left\{I_{i} \mid i \in Q_{0}\right\}
$$

is a complete set of indecomposable injective objects in $\operatorname{rep}(Q)$.

It is worth mentioning that $\operatorname{rep}(Q)$ may not contain enough projective objects. Indeed, we have the following characterization.

Proposition 6.10. The following statements are equivalent.

(1) $\operatorname{rep}(Q)$ contains enough projective objects.

(2) Every object in $\operatorname{rep}(Q)$ admits a projective cover.

(3) Every vertex in $Q$ admits only finitely many predecessors.

Proof. $(1) \Rightarrow(3)$. Assume that a vertex $b$ admits infinitely many predecessors $a_{i}$ for $i \in \Lambda$. Then there are no projective objects $P$ in $\operatorname{rep}(Q)$ with some epimorphism $P \rightarrow \bigoplus_{i \in \Lambda} S_{a_{i}}$. Indeed, otherwise each $P_{a_{i}}$ is a direct summand of $P$. Then $P(b)$ is not finite dimensional, which is a contradiction.

$(3) \Rightarrow(2)$. Assume every vertex in $Q$ admits only finitely many predecessors. In particular, $Q$ contains no right infinite paths.

Let $M$ be a representation in $\operatorname{rep}(Q)$. Assume top $M \simeq \bigoplus_{i \in \Lambda} S_{a_{i}}$ for some index set $\Lambda$. Then we obtain the following commutative diagram

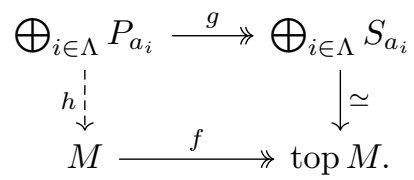

By the assumption, at most finitely many $a_{i}$ are predecessors of any given vertex $b$. Then $\bigoplus_{i \in \Lambda} P_{a_{i}}(b)$ is finite dimensional, since $Q$ is interval finite. That is to say, $\bigoplus_{i \in \Lambda} P_{a_{i}}$ lies in $\operatorname{rep}(Q)$.

We observe that $\operatorname{rad} \bigoplus_{i \in \Lambda} P_{a_{i}} \simeq \bigoplus_{i \in \Lambda} \operatorname{rad} P_{a_{i}}$. Since $Q$ contains no right infinite paths, then $f$ and $g$ are essential epimorphisms by Lemma 3.1. It follows that $h$ is an epimorphism. Moreover, it is an essential epimorphism since so is $g$. Then it is a projective cover in $\operatorname{rep}(Q)$. 
$(2) \Rightarrow(1)$. This is straightforward.

We mention that, if the equivalent conditions in the preceding proposition hold, there are no indecomposable projective objects of the form $X_{[p]}$ in $\operatorname{rep}(Q)$.

Similarly, one can prove that $\operatorname{rep}(Q)$ admits enough injective objects if and only if every vertex in $Q$ admits only finitely many successors.

\section{A Characterization for Projective objects in $\operatorname{rep}(Q)$}

Let $Q$ be an interval finite quiver. We strengthen Proposition 5.2 for countably generated flat representations in $\operatorname{Rep}(Q)$ as follows.

Lemma 7.1. A countably generated flat representation in $\operatorname{Rep}(Q)$ is a direct limit of finitely generated projective subrepresentations over the directed set $(\mathbb{N}, \leq)$.

Proof. Assume that $M$ is a countably generated flat representation. By Proposition 5.2. we may assume that $M$ is a direct limit of subrepresentations $P_{\lambda}$ lying in $\operatorname{proj}(Q)$ over some directed set $(\Lambda, \leq)$. Since $M$ is countably generated, there exist some vertices $a_{i}$ for $i \geq 0$ and some $x_{i} \in M\left(a_{i}\right)$ such that $M=\sum_{i \geq 0} M_{x_{i}}$.

We observe that for any $i \geq 0$, there exists some $\lambda_{i} \in \Lambda$ such that $x_{i} \in P_{\lambda_{i}}\left(a_{i}\right)$. Then $M_{x_{i}} \subseteq P_{\lambda_{i}}$. Let $\lambda_{0}^{\prime}=\lambda_{0}$, and choose some $\lambda_{i+1}^{\prime}$ with $\lambda_{i+1}^{\prime} \geq \lambda_{i}^{\prime}, \lambda_{i+1}$ for any $i \geq 0$ inductively. Then for any $1 \leq j \leq i$, we have that $x_{j} \in P_{\lambda_{i}^{\prime}}\left(a_{j}\right)$ and hence $M_{x_{j}} \subseteq P_{\lambda_{i}^{\prime}} \subseteq P_{\lambda_{i}^{\prime}}$. We observe that $\left(P_{\lambda_{i}^{\prime}}, \subseteq\right)$ forms a direct system over the directed set $(\mathbb{N}, \leq)$. Then $M=\sum_{i \geq 0} M_{x_{i}}=\bigcup_{i \geq 0} P_{\lambda_{i}^{\prime}}$. It implies that $M=\underline{\lim _{\longrightarrow}} P_{\lambda_{i}^{\prime}}$. Then the result follows since $P_{\lambda_{i}^{\prime}} \in \operatorname{proj}(Q)$ for any $i \geq 0$.

Then we obtain the following relationship between projective objects in $\operatorname{rep}(Q)$ and flat representations lying in $\operatorname{rep}(Q)$. This is analogous to the classical result 7 , Theorem 2.2] in module categories, which is due to [12] and [16].

Theorem 7.2. Let $Q$ be an interval finite quiver and let $M$ be a representation in $\operatorname{rep}(Q)$. Then $M$ is projective in $\operatorname{rep}(Q)$ if and only if $M$ is a direct sum of countably generated flat representations in $\operatorname{Rep}(Q)$.

Proof. For the sufficiency, we assume $M=\bigoplus_{i \in \Lambda} M_{i}$ for some indexed set $\Lambda$, such that each $M_{i}$ is a countably generated flat representation in $\operatorname{Rep}(Q)$. Lemma 7.1 implies that $M_{i}$ is a direct limit of representations lying in $\operatorname{proj}(Q)$ over $\mathbb{N}$. It follows from Lemma 6.1 that $M_{i}$ is projective in $\operatorname{rep}(Q)$. Then so is their direct sum, i.e., $M$ is projective in $\operatorname{rep}(Q)$.

For the necessity, we assume $M$ is projective in $\operatorname{rep}(Q)$. By Lemma [3.6] we have a decomposition $M=\bigoplus_{i \in \Lambda} M_{i}$ for some indexed set $\Lambda$, such that each $M_{i}$ is indecomposable. Then each $M_{i}$ is a projective object in $\operatorname{rep}(Q)$. Theorem 6.7 implies that either $M_{i} \simeq P_{a}$ for some vertex $a$ or $M_{i} \simeq X_{[p]}$ for some equivalence class $[p]$ of right infinite paths. Then the result follows since both $P_{a}$ and $X_{[p]}$ are countably generated flat representations in $\operatorname{Rep}(Q)$.

Corollary 7.3. Let $Q$ be a connected strongly locally finite quiver and let $M$ be a representation in $\operatorname{rep}(Q)$. Then $M$ is projective in $\operatorname{rep}(Q)$ if and only if $M$ is a flat representation in $\operatorname{Rep}(Q)$.

Proof. We observe that a connected strongly locally finite quiver is countable. Then each pointwise finite dimensional representation of $Q$ is countably generated. We mention that flat representations in $\operatorname{Rep}(Q)$ are closed under direct sums. Then the result is a direct consequence of Theorem 7.2 


\section{ACKNOWLEDGEMENTS}

The author is very grateful to Professor Xiao-Wu Chen for his encouragement and many helpful suggestions. The author also thanks Professor Yu Ye and Professor Shiping Liu for some suggestions, and thanks Doctor Zhe Han, Doctor Bo Hou and Doctor Dawei Shen for some discussions. He would like to thank the referee for many helpful suggestions and comments.

\section{REFERENCES}

[1] F. W. Anderson and K. R. Fuller, Rings and categories of modules, Graduate Texts in Mathematics, vol. 13, Springer-Verlag, New York-Heidelberg, 1974.

[2] L. Angeleri Hügel and J. A. de la Peña, Locally finitely generated modules over rings with enough idempotents, J. Algebra Appl. 8 (2009), 885-901.

[3] R. Bautista and S. Liu, The bounded derived categories of an algebra with radical squared zero, J. Algebra 482 (2017), 303-345.

[4] R. Bautista, S. Liu, and C. Paquette, Representation theory of strongly locally finite quivers, Proc. Lond. Math. Soc. (3) 106 (2013), 97-162.

[5] K. Bongartz and P. Gabriel, Covering spaces in representation-theory, Invent. Math. 65 (1982), 331-378.

[6] X.-W. Chen, Irreducible representations of Leavitt path algebras, Forum Math. 27 (2015), $549-574$.

[7] V. Drinfeld, Infinite-dimensional vector bundles in algebraic geometry: an introduction, The unity of mathematics, Progress in Mathematics, vol. 244, Birkhäuser Boston, Boston, MA, 2006, pp. 263-304.

[8] P. Gabriel, The universal cover of a representation-finite algebra, Representations of algebras (Puebla, 1980), Lecture Notes in Mathematics, vol. 903, Springer, Berlin-New York, 1981, pp. 68-105.

[9] P. Gabriel and A. V. Roiter, Representations of finite-dimensional algebras, Algebra VIII, Encyclopaedia of Mathematical Sciences, vol. 73, Springer, Berlin, 1992, pp. 1-177.

[10] V. E. Govorov, On flat modules, Sibirsk. Mat. Ž. 6 (1965), 300-304.

[11] P. Jiao, Decomposition of pointwise finite length modules over an essentially small category, preprint, arXiv:1807.05668.

[12] I. Kaplansky, Projective modules, Ann. Math. 68 (1958), 372-377.

[13] D. Lazard, Sur les modules plats, C. R. Acad. Sci. Paris 258 (1964), 6313-6316.

[14] H. Lenzing and R. Zuazua, Auslander-Reiten duality for abelian categories, Bol. Soc. Mat. Mexicana (3) 10 (2004), 169-177.

[15] U. Oberst and H. Röhrl, Flat and coherent functors, J. Algebra 14 (1970), 91-105.

[16] M. Raynaud and L. Gruson, Critères de platitude et de projectivité, Invent. Math. 13 (1971), $1-89$.

[17] J. J. Rotman, An introduction to homological algebra, Universitext, Springer, New York, second ed., 2009

[18] S. P. Smith, The space of Penrose tilings and the noncommutative curve with homogeneous coordinate ring $k\langle x, y\rangle /\left(y^{2}\right)$, J. Noncommut. Geom. 8 (2014), 541-586.

[19] C. A. Weibel, An introduction to homological algebra, Cambridge Studies in Advanced Mathematics, vol. 38, Cambridge University Press, Cambridge, 1994.

Department of Mathematics, China Jiliang University, Hangzhou 310018, PR China

E-mail address: jiaopjie@cjlu.edu.cn 